\begin{tabular}{lllll}
\hline Estudios de Economía Aplicada & Vol. 36-2 & 2018 & PÁgs. 429-458 \\
\hline
\end{tabular}

\title{
La participación en el “due process” del IASB*
}

\author{
Horacio Molina SÁNCHEZ ${ }^{\text {a }}$, RAFAel BaUtista MEsa ${ }^{\text {a }}$ \\ a Universidad Loyola Andalucía, Facultad de Ciencias Económicas y Empresariales. C/Escritor \\ castilla Aguayo, 4.14004 Córdoba. E-mail: hmolina@uloyola.es; rbautista@uloyola.es
}

\begin{abstract}
RESUMEN
Los procesos de consulta abierta son un mecanismo por el cual las organizaciones privadas internacionales tratan de buscar la legitimidad de sus actuaciones. Esta legitimidad en el input se considera crítica y ha dado lugar a la puesta en marcha de acciones tendentes a dinamizarla. Nuestros resultados han puesto de manifiesto que existe un grupo de entidades que asumen un compromiso de participación recurrente en el IASB. Esta participación dota de legitimidad a sus pronunciamientos. Estas entidades son las referencias en conocimiento experto en el ámbito de la emisión de normas contables nacionales y en el ámbito de la práctica de auditoría.
\end{abstract}

Palabras clave: IASB, Lobbying, Proceso debido, Regulación contable.

\section{Participation in the IASB Due Process}

\begin{abstract}
Open consultations are mechanisms by which private international organizations try to gain legitimacy. This legitimacy in the input is considered critical and has given rise to the implementation of actions aimed at enhancing it. Our results show a group of entities that assume a commitment to recurring participation in the IASB. This participation gives legitimacy to IASB's pronouncements. These entities are reference points with expert knowledge in the field of the issuance of national accounting standards and in the field of auditing.
\end{abstract}

Keywords: IASB, Accounting Lobbying, Due process, Accounting regulation.

Clasificación JEL : M41, M48

\footnotetext{
${ }^{*}$ Este trabajo ha sido parcialmente financiado dentro del Programa Ayudas para la financiación de actividades de investigación a grupos de investigación (SEJ425) de la Junta de Andalucía.
}

Artículo recibido en marzo de 2018 y aceptado en abril de 2018

Artículo disponible en versión electrónica en la página www.revista-eea.net, ref. ə-36209 


\section{INTRODUCCIÓN}

La globalización de las finanzas ha exigido dar respuestas globales, más allá de las jurisdicciones nacionales, para regular las instituciones que favorecen el desarrollo de los mercados financieros internacionales. Entre ellos, la armonización contable internacional es un reto que facilita la asignación de recursos a nivel global.

La armonización contable internacional, que se inicia en torno a los trabajos del International Accounting Standard Committee (IASC) y, posteriormente, su metamorfosis en el International Accounting Standard Board (IASB) es una realidad con amplia aceptación internacional ${ }^{1}$. El IASB es el órgano de gestión de la Fundación IFRS, encargado por tanto del desarrollo de su misión, donde juega un papel crucial la emisión y revisión de las International Financial Reporting Standards (IFRS). La Fundación IFRS es una organización privada y, por tanto, ha de desarrollar mecanismos de legitimación de su actividad que den respuesta a las siguientes cuestiones: ¿por qué las normas emitidas por el IASB se deben aceptar? Dado que ningún ciudadano ha elegido a sus miembros para que les represente, ¿garantizan sus procedimientos que sus trabajos redunden en el bienestar de la sociedad, evitando las presiones de los grupos de interés?, ¿ante quiénes rinden cuentan? o ¿quién supervisa su actuación?

La legitimidad en la emisión de normas contables de carácter internacional reside en última instancia en los Estados (o en instituciones supranacionales cuando los Estados han delegado estas competencias en ellos como, por ejemplo, la Unión Europea); sin embargo, una vez se decide la aplicación de las normas internacionales en un ámbito jurisdiccional, aunque sea con mecanismos de aceptación (el endorsement en el ámbito de la Unión Europea), se produce de facto una cesión de competencias. Es más, una vez que las normas internacionales hayan ganado un grado elevado de aceptación resulta muy difícil que una jurisdicción pueda evitar su adopción, por motivos de competitividad, acceso a mercados de financiación o participación en los programas de otros organismos multilaterales (Richardson y Everlyn, 2011). Los esquemas democráticos parecen encajar con dificultad en el gobierno de las relaciones globales y aquí es donde una tercera vía propuesta por Richardson y Everlyn (2011) pasa por reforzar la legitimidad de las instituciones mediante la construcción de modelos de buen gobierno de estas instituciones para la globalización.

Entre esas fuentes de legitimidad, unas van destinadas a legitimar al sujeto emisor de normas, el quién. El sujeto ha de reunir dos características básicas: competencia técnica e independencia. El IASC, desde su origen, fue un organismo donde primaba en la elección el carácter representativo, coherente con su estrategia primigenia de "ganar aceptación" (Camfferman y Zeff, 2007).

\footnotetext{
${ }^{1}$ El cambio de denominación del IASC a IASB tuvo lugar en 2001.
} 
Posteriormente, en 1989 inicia una etapa de mejora de la calidad de las normas, sin abandonar completamente la dimensión política de sumar voluntades. Cuando recibe el apoyo del IOSCO en 1995, cumple las condiciones sugeridas por el organismo mundial que agrupa a los supervisores de mercados de valores, alcanzando el objetivo de generar adhesión. En ese momento, la estrategia pone a la calidad técnica en el centro de la agenda y la representación pasa a ser un adjetivo. La primera constitución del IASB, pone el acento en la capacidad técnica de sus miembros y en su independencia respecto a las entidades de procedencia. Posteriormente, la constitución ha sido reformada en varias ocasiones, reforzando el buen gobierno de la Fundación IFRS. En este periodo se han mejorado aspectos relacionados con la gobernanza como son: la separación de funciones entre gestión (en el consejo) y gobierno (en el patronato) para mitigar las influencias indebidas, el establecimiento de mecanismos de selección de miembros que aporte diversidad (en cuanto a procedencias geográficas y profesionales) y la creación de órganos consultivos con amplia representación.

Una segunda fuente de legitimidad es de carácter procedimental, el cómo, y su objetivo es triple: ofrecer transparencia a los interesados, que el organismo reciba los inputs de calidad que precisa y generar adhesión a través de la participación. Las cuestiones claves son cómo incorporar un tema a la agenda, cómo se delibera durante el proceso de formulación de la norma y cómo se aprueba, buscando mayorías reforzadas que implican niveles próximos al consenso. Este mecanismo de legitimidad procedimental se denomina due process.

La tercera fuente, denominada por Burlaud y Colasse (2011) como legitimidad sustancial, procura la calidad técnica de las normas contables, el qué de la regulación contable y se fundamenta sobre la consistencia de los pronunciamientos con el marco conceptual. Esta referencia a unos criterios generales que orientan la labor normalizadora, unida a la competencia técnica de los miembros del IASB, dota de legitimidad técnica a las normas emitidas.

Este trabajo indaga sobre los atributos que garantizan la legitimidad procedimental. Richardson y Eberlyn (2011) distinguen una legitimidad del input, que se obtiene con una amplia participación; una legitimidad en el proceso, que se fundamenta en el cómo se llega a la norma y que, en el caso de organismos transnacionales, ha de basarse en procesos deliberativos; una tercera, la legitimidad del output, que se sostiene en una elevada calidad técnica de los pronunciamientos. La importancia del due process responde, según Richardson y Eberlyn (2011), a tres razones: un cambio del gobierno en el IASB frente al IASC, pasando de un carácter representativo a uno técnico; el deseo de implantar las normas en las jurisdicciones sin modificaciones y evitar que surgiesen organismos internacionales alternativos. El due process aporta dos ventajas adicionales a la legitimidad: por un lado, ofrece un método sistemático para resolver problemas, al permitir identificar un problema contable, las cuestiones 
subyacentes, las posibles alternativas, evaluar entre alternativas y decidir las mejores (Miller, Redding y Bahnson, 1994); por otro, abre un espacio para expresar las reservas que puedan tener los interesados que posteriormente deben aplicarlas; es decir, se pretende facilitar la aceptación de estas normas (Tandy y Wilburn, 1992).

En los últimos años se ha desarrollado una amplia investigación sobre la legitimidades del input; así, se indaga en quiénes participan en el proceso (Larson, 2007; Georgiou, 2010; Durocher y Fortin, 2011; Jorissen et al., 2012, 2013 y 2014; Larson y Hertz, 2013; Procházka, 2015; Dobler y Knospe, 2016b; Mellado y Parte, 2017; Eisenschmidt y Krasodomska, 2017) o cómo argumentan (Giner, y Arce, 2012; Molina y Mora, 2015) y sobre la legitimidad del proceso, estudiando quién influye (Procházka, 2015; Dobler y Knospe, 2016a; Bamber y McMeeking, 2016).

Esta literatura toma sus precedentes en los trabajos sobre lobbying y en los marcos teóricos desarrollados para explicar el comportamiento de los participantes en el proceso. Siguiendo a Durocher et al. (2007), la explicación de los modelos de participación se ha soportado en varias teorías: la teoría positiva de la contabilidad, siendo el propio interés el que explica la participación. Otra línea argumental se ha fundamentado en la teoría económica de la democracia. Según ésta, la participación viene determinada por el beneficio del participante y por su capacidad de influir (Sutton, 1984). Un último modelo de explicación, el Coalition and Influence Group, ha tratado de identificar coaliciones de grupos de interés y su influencia en el emisor de normas. Königsgruber (2014) propone un modelo analítico del lobbying según el cual el participante suministra información relevante para el organismo emisor de normas. Este marco de relación entre participantes y organismo emisor estaría en consonancia con modelos procedentes de la ciencia política que atribuyen al proceso de lobbying el valor de suministrar información creíble.

Nuestra investigación pretende explicar el fenómeno de la participación formal en el due process considerando el comportamiento individual de cada participante en un periodo prolongado de tiempo. Esta dimensión que informa sobre la intensidad en el lobbying ha sido abordada por la literatura en los documentos emitidos en un proyecto concreto (Kosi y Reither, 2014, para el caso de la reforma de la contabilidad de los seguros; Mellado y Parte, 2017, en el caso de la reforma de la norma de arrendamientos) pero no con todos los proyectos en un periodo. El análisis de la intensidad en la participación, con un enfoque multi-periodo y multi-proyecto, sólo hemos encontrado el estudio sobre preparadores de Jorissen et al. (2012). Nuestros resultados muestran una red de participantes que colaboran regularmente aportando su visión, a favor o en contra, a las diversas propuestas del IASB. 
Nuestros resultados permiten afirmar que las firmas de auditoría mundiales y algunos emisores de normas nacionales, así como algunas asociaciones de auditores son los más comprometidos con la labor del IASB y sus principales legitimadores. Por el contrario, los preparadores no tienen una implicación continuada en los proyectos del IASB.

El siguiente epígrafe lo destinamos a la revisión de la literatura y el desarrollo de hipótesis. El epígrafe tercero describe la metodología y la captura de datos. El capítulo cuarto se destina a presentar los resultados y su discusión. Finalmente, el punto quinto lo destinamos a las conclusiones.

\section{REVISIÓN DE LA LITERATURA Y DESARROLLO DE HIPÓTESIS}

La participación en el due process dota de legitimidad a los pronunciamientos del IASB. Esta legitimidad en el input del proceso de emisión de normas se ha estudiado en función de varios atributos como son: el número de cartas recibidas por el IASB, la diversidad geográfica de los remitentes y la diversidad de colectivos implicados en la información financiera. Jorissen et al. (2013) y Larson y Herz (2013) estudian respectivamente, en el horizonte temporal 1995 a 2007 y 2001 a 2008, el número de cartas de los distintos colectivos en función de su procedencia geográfica. Más recientemente, Dobler y Knosper (2016) han estudiado el periodo 2006-2012.

En cuanto al número de cartas remitidas, los resultados de los dos primeros estudios ponen de manifiesto que el IASB ha incrementado su legitimidad habiendo recibido tras la reforma del año 2001 una media en torno a 100 cartas por proyecto ${ }^{2}$. En el periodo 2006 a 2010, Dobler y Knosper (2016) documentan una media de 157,59 cartas por proyecto. Estos datos suponen un crecimiento en el número de cartas recibidas si se compara con las recibidas por su predecesor el IASC y de las recibidas por los comités de interpretación de normas (Larson y Herz, 2013) y superando también las 134 cartas que recibía de media el FASB en sus primeros 100 proyectos (Tilburn, 2013).

La diversidad geográfica también incrementó con la transformación del IASC en el IASB, especialmente por la incorporación de países en vías de

\footnotetext{
2 Jorissen et al. (2013) observaron para una submuestra de 2001 a 2007 y 45 proyectos, 100,71 cartas, mientras que Larson y Herz (2013) obtuvieron para el periodo 2001 a 2008 y 57 proyectos una media de 104 cartas por proyecto. Este periodo viene caracterizado por la reforma de la Institución, la decisión de adopción de la Unión Europea mediante el Reglamento 1606/2002 por el que las cuentas anuales consolidadas de los grupos cotizados a partir de enero de 2005 debían presentarse bajo Norma Internacionales de Información Financiera y por la firma en 2002 del Acuerdo de Norwalk con el FASB para propiciar la convergencia entre las normas del IASB y el FASB, dando como resultado la firma del Memorandum of Understanding en 2006, donde se establece una agenda conjunta hacia la convergencia.
} 
desarrollo (Jorissen et al., 2013 y Larson y Herz, 2013). Larson y Herz (2013) explican que la participación (tanto en cartas enviadas como en participantes) por origen geográfico se encuentra en la pertenencia a la Unión Europea, al G4+1, ser donante del IASB y tener un mercado de valores más desarrollado (Dobler y Knospe, 2016).

La tradición regulatoria de un país puede ejercer una influencia en el comportamiento de los participantes. A través de una encuesta en dos países con tradiciones diferentes, Orens et al. (2011) observan que los preparadores de contextos diferentes tienen diferentes métodos de participación (directamente o a través del auditor), percepciones diferentes sobre la eficacia de su acción y las razones distintas que le llevan a participar. Jorissen et al. (2013), estudiando los datos de participación en el IASB, observan también que la diferencia en los marcos institucionales y los costes de participación influyen en el nivel de participación. Así, los preparadores participan, en mayor medida, cuando en el país existen sólidos mecanismos ex post de vigencia efectiva de la norma, mientras que los no preparadores de países con elevados niveles de protección ex ante a los inversores muestran niveles superiores de participación (a la misma conclusión llega con su modelo analítico Königsgruber, 2013). Por otra parte, considerando los costes de participación, la familiaridad con procesos de emisión de normas en entidades privadas, la congruencia con los valores del IASB (transparencia, neutralidad en la valoración y juicio profesional) o el idioma influyen positivamente en las cartas enviadas (Dobler y Knospe, 2016). Los factores culturales también influyen en el grado de participación. Así, el grado de individualismo en una sociedad, medido según la escala de Hofstede, propicia una mayor participación de los individuos en los sistemas políticos y se ha observado que el grado de individualismo se relaciona positivamente con la participación en el due process del IASB (Dobler y Knospe, 2016). Sin embargo, la adopción de las normas por una jurisdicción no influye en una mayor participación desde esos países (Jorissen et al. 2013; Larson y Herz, 2013; Dobler y Knospe, 2016).

Estos resultados alertan que la participación se encuentra limitada mientras no se produzca un mayor grado de convergencia en los entornos institucionales; mientras tanto es preciso proponer medidas alternativas que permitan ganar legitimidad (Jorissen et al., 2013; Larson y Herz, 2013).

Por su parte, respecto a la diversidad de colectivos participantes, los preparadores son el colectivo que más cartas ha enviado al IASB desde la reforma del IASB en 2001. Este colectivo representaba el 39\% del total de cartas remitidas, seguido de la profesión auditora con un 29,5\%. El tercer colectivo importante son los emisores de normas contables nacionales que suponen un $17,7 \%$ del total. Entre estos tres colectivos suponen cerca del $90 \%$ de las cartas 
desde 2001 (Jorissen et al., 2013) ${ }^{3}$. El resto de stakeholders relacionados con la información financiera tiene una participación muy escasa, siendo motivo de preocupación la escasa implicación de los usuarios. La reforma de 2001 y la adopción generalizada de las normas internacionales en las distintas jurisdicciones (especialmente para sus compañías cotizadas en mercados de valores) no modificaron la hegemonía de estos tres grupos; sin embargo, trajo consigo un aumento de la proporción de cartas de los emisores de normas nacionales y una menor proporción de cartas de la profesión auditora. Por su parte, los preparadores de mayor dimensión y más rentables son los que presentan mayores niveles de participación (Jorissen et al., 2012).

La escasa participación de los usuarios se observa en los estudios que han cuantificado las cartas remitidas al IASB (Jorissen et al., 2013 y Larson y Hertz, 2013, Dobler y Knospe, 2016). El coste de participación es la principal razón que lleva a no participar directamente, por lo que suele ser frecuente hacerlo a través de las asociaciones profesionales. El coste y la carencia de recursos intelectuales son dos barreras importantes a la participación como apuntan Burlaud y Colasse (2011); de hecho, las empresas que participan en los proyectos son de mayor dimensión y más rentables que las que no lo hacen y las empresas que participan más habitualmente son las de mayor dimensión (Jorissen et al., 2012).

Las características del proyecto objeto de consulta influyen en el nivel de participación de los diversos colectivos. Así, Jorissen et al. (2012) muestran que la participación de la profesión auditora, los emisores de normas y los académicos se centra en las etapas más tempranas del proceso de emisión de una norma (discussion paper), mientras que los preparadores se concentran más en las fases finales (exposure draft). La participación aumenta conforme avanza el desarrollo de un proyecto (Procházka, 2015 ${ }^{4}$ ). Otro factor que explica la participación es el número de preguntas; así, los proyectos con más preguntas presentan niveles de participación más elevados pues se presentan un mayor número de oportunidades para emitir un comentario (Dobler y Knospe, 2016). Los proyectos con mayor impacto en las cifras de una compañía provocan una participación más elevada de preparadores, profesión auditora y emisores de normas (Jorissen et al., 2012). Sin embargo, la evidencia sobre el grado de participación en los proyectos centrados en la revelación no es concluyente (Jorissen et al., 2012 y Dobler y Knospe, 2016). Asimismo, el nivel de controversia de un proyecto, observable cuando el proyecto es revisado o es

\footnotetext{
${ }^{3}$ Un dato significativo es la menor proporción de cartas procedentes de la profesión de auditoría en el periodo anterior a la reforma y el posterior, con una caída del $40 \%$ del total de cartas al $29,1 \%$.

${ }^{4}$ El estudio contiene los proyectos emitidos por el IASB entre 2006 y 2014.
} 
retirado por el IASB, incrementa el nivel de participación y pone de manifiesto que la participación ejerce influencia en el IASB (Procházka, 2015).

La participación indirecta ha sido estudiada para el caso del European Financial Reporting Advisory Group (EFRAG) el cual tiene abierto un proceso de consulta formal previo a emitir su posicionamiento ante el IASB (Jorissen et al., 2012). Los resultados que han obtenido revelan que los participantes optan fundamentalmente por la participación directa. La participación indirecta, en el caso europeo a través del EFRAG, la realizaron fundamentalmente emisores de normas nacionales y los supervisores de mercados de valores. Otra forma de participación indirecta es a través de asociaciones profesionales (Georgiou, 2010) o a través de los auditores de la compañía (Orens et al., 2011; sin embargo, este comportamiento no ha sido observado por Jorissen et al., 2006, ni MacArthur, 2012).

Nuestro trabajo pretende indagar en el marco de compromiso de los participantes con el IASB en su misión de emisor mundial de normas contables. Para ello, nos fundamentamos en el marco analítico propuesto por Königsgruber (2013) y que configura el due process como una fase de colaboración entre el IASB y los grupos de interés, formulado por Miller, Redding y Bahnson (1994) para el FASB. En este marco analítico, las partes interesadas participan para aportar su conocimiento experto. Este modelo propone que la participación pretende aportar información creíble. La participación habitual en el due process sirve para señalizar que se dispone de un conocimiento experto, creando esa expectativa en el decisor.

Bajo este marco teórico deducimos que las firmas internacionales de auditoría y las asociaciones de auditores, por su amplio conocimiento de las problemáticas abordadas, son una fuente relevante de información para conocer los fundamentos de las cuestiones tratadas, proponer alternativas posibles y formular soluciones a las mismas. La armonización contable internacional produce economías de escala en las firmas internacionales de auditoría pues el departamento técnico se especializa en un único marco de información. De hecho, el compromiso de las firmas internacionales de auditoría va más allá de la legitimidad en los inputs, abarcando otras esferas como la colaboración de profesionales en los equipos técnicos del IASB y la contribución financiera al sostenimiento de la Fundación IFRS. En el ámbito del IASB, este resultado ha sido corroborado en trabajos previos (Jorissen et al., 2013; Larson y Hertz, 2013, Dobler y Knosper, 2016)

H1a. Las firmas de auditoría internacionales presentan un nivel de participación superior que el resto de participantes

H1b. Las asociaciones de auditores presentan un nivel de participación superior que el resto de participantes

La adopción de las normas internacionales de información financiera supone una pérdida de soberanía para el emisor de normas contables del país. Su papel 
pasa a tener un carácter proactivo en la emisión de normas. De hecho, en la construcción del esquema de endorsement de las normas internacionales de contabilidad en Europa, el EFRAG asume, por un lado, un papel asesor en el endorsement de una norma por la Comisión Europea y el Comité de Regulación Contable y, por otro, una función de actuación proactiva en el due process del IASB. La participación de estos emisores de normas permite hacer llegar los efectos que determinadas propuestas pueden tener en contextos internacionales que son diversos. Por otra parte, estos emisores de normas nacionales son las instituciones que han cedido sus competencias al IASB para determinadas empresas, reteniendo las competencias normativas para otras. Estos participantes deben equilibrar las particulares necesidades de las entidades que se negocian en mercados de valores con las de otras entidades que no cotizan y, al mismo tiempo, evitar grandes diferencias entre los marcos de información que conviven en su jurisdicción. Nuestra hipótesis 2 es que este colectivo es uno de los que mantiene una implicación elevada con el IASB porque disponen de conocimiento técnico, disponen de información específica del contexto nacional que puede tener interés para la redacción de la norma y comparten la misión de emitir normas en interés de los usuarios de la información.

H2. Los emisores de normas contables muestran un nivel de implicación superior que otros participantes

Por el contrario, el comportamiento de los preparadores se ha explicado en función de su interés bajo el marco formulado por Sutton (1984). Su participación ha de ser más esporádica porque sólo intervienen cuando la norma tiene un efecto en sus estados financieros y la capacidad de influencia sea elevada. Bajo el modelo analítico de Königsgruber (2013), el participante puede generar una relación de confianza con el emisor de normas en la que permita señalar que no posee información en todos los proyectos.

H3. Los preparadores muestran un nivel de implicación inferior que otros participantes

La procedencia geográfica y el entorno institucional han sido analizadas en otros trabajos como variables explicativas del nivel de participación. Las incorporamos a nuestros modelos como variables de control.

\section{DATOS Y METODOLOGÍA}

La toma de datos procede de la página web del IASB. En ella figuran los proyectos y las cartas de comentario. Los datos han sido tabulados considerando como unidad de dato cada participante y como observaciones su participación en cada uno de los proyectos. El periodo temporal que abarcan nuestras observaciones es de 12 años entre 2004 y 2015. Nuestra base de datos consta de 
132 proyectos sobre un total de 134 proyectos $^{5}$, lo que representa un $98,5 \%$ de cobertura. El número de cartas enviadas asciende a 17.077, con un total 3.879 participantes ${ }^{6}$.

Los estudios realizados hasta la fecha sobre las cartas de comentario han tomado fundamentalmente como unidad de muestreo la carta de comentario. Tan sólo Jorissen et al. (2012) realizan un análisis del grado de implicación (al que denominan "lobbying intensity") de las empresas participantes en 22 proyectos para estudiar si viene explicado por variables económicas de la entidad. Nuestra unidad de muestreo es similar, el participante, pero la extendemos más allá del colectivo de preparadores y para un volumen de proyectos más representativo. Esta dimensión permite superar la debilidad que presentan los estudios que analizan como unidad de muestreo la carta individual pueda suponer la incorporación de proyectos con gran número de intervenciones, aisladas a ese proyecto, y con escaso contenido informativo.

Asimismo, esta dimensión individual es necesaria si el objetivo es medir el grado de implicación de los participantes, dado que el número de cartas de un colectivo no ofrece información sobre el comportamiento individual. Por tanto, este estudio contribuye a otros anteriores pues tiene en consideración la dimensión individual del participante en un contexto multi-periodo y multiproyecto.

Las cartas identificadas fueron asignadas al país de procedencia y el colectivo de pertenencia. Cuando la respuesta es colectiva, lo hemos tratado como un participante diferente de los miembros que escriben conjuntamente. Esta decisión minora en algún caso el nivel de participación si el participante opta por efectuar algunas intervenciones individualmente y otras conjuntamente. Por otra parte, cuando el participante ha cambiado de denominación se ha agregado su participación a la de la denominación anterior (por ejemplo, el Accounting Standard Board británico cambió a Financial Reporting Council).

La asignación a país de procedencia en el caso de los organismos internacionales (por ejemplo, el IOSCO, Comité de Basilea), de las firmas internacionales de auditoría o respuestas conjuntas con varios países de procedencia fue "Internacional” como una categoría adicional. En el caso de las

${ }^{5}$ Los proyectos que no hemos podido recabar las cartas de comentario son: ED 2006. Amendments to IFRS 6 Exploration for and Evaluation of Mineral Resources and IFRS1 Firsttime Adoption of IFRSs y la Review of the Constitution de 2008. Public Accountability and the Composition of the IASB. Proposals for Change, part 1.

${ }^{6}$ Nuestro trabajo recoge la base de datos más amplia de aquéllos que han analizado desde una perspectiva multi-periodo y multi-proyecto la participación en el IASB. Jorissen et al. (2013) analizaron 7.442 cartas, Larson y Hertz (2013) recogieron 5.785 cartas y Dobler y Knospe (2013) 8.825. 
empresas u ONGs multinacionales asignamos el país donde radica la sede social.

La asignación al colectivo de pertenencia se dedujo de la etiqueta del IASB cuando existía, la información que se derivaba de la propia carta de comentario o finalmente de una búsqueda por internet. En algunos casos hemos tenido que proceder a asignarlo a la etiqueta de "desconocido" que hemos eliminado de nuestro análisis. Esta depuración nos deja una base de datos para efectuar nuestros análisis de 15.951 cartas (94,33\% del total) y 3.672 participantes (94,66\% del total).

La clasificación de colectivos se fundamenta en las identificaciones realizadas por el IFAC (2008) como interesados en la cadena de información, en otros trabajos (Jorissen et al., 2013; Larson y Hertz, 2013, Dobler y Knosper, 2016) y en desgloses adicionales para recoger la participación, por ejemplo, de estudiantes, ONGs o abogados. Existen colectivos que pueden tener un doble rol, por ejemplo, las entidades financieras o de seguro, bien actuando como preparadores, bien como usuarios de estados financieros cuando deciden asignar créditos o realizar inversiones.

El contraste de hipótesis lo realizamos con un análisis de la varianza en la que el factor es la pertenencia o no a cada uno de los colectivos recogidos en nuestras hipótesis. Así, EMI es una variable dicotómica que toma el valor 1 si es un emisor de normas contables y 0 en otro caso. PREP toma el valor si el participante es preparador de la información. ASOCAUD toma el valor 1 si el participante es una asociación o regulador de auditores. La variable FIRMAUD queda excluida del análisis multivariante por su distorsión sobre el modelo de regresión lineal; en este sentido, consideramos casos especiales los 10 sujetos del total de la muestra que toman el valor 1 como firma de auditoría internacional, puesto que presentan una media de 93,0 cartas enviadas frente a las 4,3 cartas de media para el total de la muestra.

Las variables de control que vamos a utilizar se relacionan o con la procedencia geográfica o con la tradición legal. En cuanto a la procedencia geográfica, diversos estudios han puesto de manifiesto la sobrerrepresentación de cartas procedentes de Europa (Jorissen et al., 2013 y Larson y Herz, 2013); por este motivo definimos la variable EUR, que toma el valor 1 cuando el participante procede de Europa y 0 en otro caso. En cuanto a la tradición legal, se argumenta que los países del ámbito anglosajón están más familiarizados con el modelo de regulación privado y se ha probado que induce a una respuesta superior (Orens et al., 2011, Jorissen et al., 2013, Dobler y Knospe, 2016). A tal fin, utilizamos la clasificación de La Porta et al. (1998), tomando ANGLO el valor 1 si el participante procede de un país con tradición jurídica anglosajona y 0 en otro caso. 
Adicionalmente, formulamos un modelo de regresión para evaluar en qué medida cada colectivo tiene una mayor participación. El modelo se formula:

$$
\text { CarPar }=\alpha+\beta_{1} E M I+\beta_{3} P R E P+\beta_{3} A S O C A U D+\beta_{4} E U R++\beta_{5} A N G L O+\varepsilon
$$

La variable dependiente CarPar representa el número de cartas por participante. Utilizaremos también CarPar1 en la que cuantificamos la variable con los proyectos respondidos entre 2004 y 2010 y CarPar2 para la que contamos los proyectos entre 2011 y 2015.

\section{ANÁLISIS DE RESULTADOS}

El epígrafe de resultados lo dividimos en los resultados descriptivos, a continuación, el contraste de las hipótesis y finalmente unos análisis adicionales que complementan la información sobre la participación de cada sujeto.

\subsection{Descriptivos}

Los resultados muestran un número medio de cartas por proyecto de 129,37 cartas. Este resultado se sitúa en un punto intermedio entre los 157,59 obtenidos por Dobler y Knosper (2016) y los 100,71 de Jorissen et al. (2013) y 104 de Larson y Herz (2013). El ratio de Dobler y Knosper (2016) está influido por la importancia de algunos proyectos $\mathrm{MoU}$, mientras que en nuestro estudio, tras esos proyectos se ha vuelto a niveles más moderados de participación. En cuanto a la dimensión de la legitimidad del input fundamentada en el número de cartas recibidas, estos resultados muestran que el IASB debe seguir buscando mecanismos que aumenten la participación.

Tabla 1

Distribución de participantes por origen geográfico en 132 proyectos del IASB entre 2004-2015

\begin{tabular}{|lcc|}
\hline \multicolumn{1}{|c}{ Procedencia geográfica } & \% sujetos & \% cartas \\
\hline Asia y Oceanía & $15,99 \%$ & $19,91 \%$ \\
Europa & $32,75 \%$ & $41,88 \%$ \\
África & $3,67 \%$ & $3,30 \%$ \\
Norteamérica & $41,24 \%$ & $21,10 \%$ \\
Latinoamérica & $3,37 \%$ & $2,99 \%$ \\
Mundial & $2,98 \%$ & $10,81 \%$ \\
Número total & 3.652 & 15.933 \\
\hline
\end{tabular}

Fuente: Fundación IFRS y elaboración propia.

La Tabla 1 presenta los porcentajes de sujetos que participan y cartas enviadas en el due process por origen geográfico. El mayor número de participantes procede de Norteamérica, seguido de Europa y en tercer lugar Asia y Oceanía. Sin embargo, el origen de las cartas es fundamentalmente de Europa seguido por Norteamérica y Asia y Oceanía con un porcentaje muy similar. Estos resultados 
se explican en gran medida por los proyectos del MoU relativos a instrumentos financieros, reconocimiento de ingresos y arrendamientos en los que el nivel de participación desde EE.UU. ha sido muy intenso y puntual.

La Tabla 2 muestra las frecuencias de participación considerando el colectivo de pertenencia. El colectivo que más participa es el de preparadores, suponiendo un 60,68\% de los participantes. El número de cartas más elevado procede de los preparadores no financieros, seguido de los emisores de normas, asociaciones de auditores, entidades financieras y las firmas internacionales de auditoría. Estos resultados son compatibles con los observados por Jorissen et al. (2013) y por Dobler y Knosper (2016) que situaban a este colectivo en el 39\%; la suma de las cartas de los preparadores no financieros, las entidades bancarias y las entidades de seguros asciende al 43,3\%. Se aprecia, por otra parte, una minoración de la importancia de la profesión auditora que se encuentra en el $22 \%$, frente al $29 \%$ del estudio de Jorissen et al. (2013).

Tabla 2

Distribución de participantes por origen geográfico en 132 proyectos del IASB entre 2004-2015

\begin{tabular}{|lcr|}
\hline \multicolumn{1}{c}{ Colectivo } & \% sujetos & $\%$ cartas \\
\hline Emisor de normas & $1,86 \%$ & $14,33 \%$ \\
Preparadores & $60,68 \%$ & $43,53 \%$ \\
$\quad$ Preparador no financiero & $46,56 \%$ & $28,05 \%$ \\
$\quad$ Entidad financiera & $7,19 \%$ & $10,00 \%$ \\
Entidad de seguros & $6,94 \%$ & $5,47 \%$ \\
Regulador o asociación de auditores & $4,34 \%$ & $13,69 \%$ \\
Firma mundial de auditoría & $0,27 \%$ & $5,59 \%$ \\
Firma local de auditoría & $6,28 \%$ & $2,50 \%$ \\
Auditor del sector público & $0,38 \%$ & $0,81 \%$ \\
Firma mundial (opinión personal) & $0,52 \%$ & $0,16 \%$ \\
Supervisor de mercado de valores & $1,07 \%$ & $2,10 \%$ \\
Académico & $5,38 \%$ & $2,22 \%$ \\
Inversores & $6,15 \%$ & $3,86 \%$ \\
ONGs & $3,44 \%$ & $0,93 \%$ \\
Gobierno & $1,72 \%$ & $1,51 \%$ \\
Supervisor bancario y de seguros & $1,20 \%$ & $1,41 \%$ \\
Sindicatos & $0,71 \%$ & $0,17 \%$ \\
Valoradores & $2,76 \%$ & $2,23 \%$ \\
Estudiantes & $2,70 \%$ & $0,62 \%$ \\
Abogados & $0,52 \%$ & $0,11 \%$ \\
\hline TOTAL & 3.660 & 16.644 \\
\hline
\end{tabular}

Fuente: Fundación IFRS y elaboración propia.

La Tabla 3 muestra el sistema legal de procedencia tanto de los participantes como de los inputs recibidos. Un 71\% de los participantes, con un $62 \%$ de los envíos realizados, procede de países con tradición legal anglosajona, lo que 
confirmaría los hallazgos que en los inputs que recibe el IASB existe un sesgo a favor de los países del common law, frente a sistemas del civil law.

Tabla 3

Distribución de participantes y cartas recibidas según la tradición jurídica del país de emisión

\begin{tabular}{|lcc|}
\hline \multicolumn{1}{|c}{ Tradición legal } & \% sujetos & \% cartas \\
\hline Anglosajón & $71,26 \%$ & $62,91 \%$ \\
Legal francesa & $13,33 \%$ & $14,87 \%$ \\
Legal germánica & $12,39 \%$ & $17,75 \%$ \\
Legal escandinava & $3,11 \%$ & $4,46 \%$ \\
\hline Número total & $\mathbf{3 . 4 1 3}$ & $\mathbf{1 3 . 3 3 4}$ \\
\hline
\end{tabular}

Fuente: Fundación IFRS y elaboración propia.

\subsection{Contraste de Hipótesis}

La Tabla 4 muestra, mediante el análisis de la varianza, la diferencia de medias en la participación de cada colectivo respecto al resto, siendo significativo en todos los casos. Las Firmas de auditoría internacionales son las que presentan una diferencia más pronunciada (confirmando nuestra hipótesis 1a), seguidas de los emisores de normas contables y las asociaciones de auditores. Por el contrario, tal y como preveíamos, la participación de los preparadores es más esporádica y el número de cartas medio por participante es inferior a la de los participantes que no son preparadores.

Tabla 4

Análisis de la Varianza de las variables dependientes con los distintos factores

\begin{tabular}{|c|c|c|c|c|c|c|c|c|c|c|c|c|}
\hline \multirow[b]{2}{*}{ Variables } & \multicolumn{2}{|c|}{ Valor medio CarPar } & \multirow[b]{2}{*}{$\mathbf{F}$} & \multirow[b]{2}{*}{ sig } & \multicolumn{2}{|c|}{ Valor medio CarPar1 } & \multirow[b]{2}{*}{$\mathbf{F}$} & \multirow[b]{2}{*}{ sig } & \multicolumn{2}{|c|}{ Valor medio CarPar2 } & \multirow[b]{2}{*}{$\mathbf{F}$} & \multirow[b]{2}{*}{ sig } \\
\hline & Pertenece & $\begin{array}{c}\text { No } \\
\text { Pertenece }\end{array}$ & & & Pertenece & $\begin{array}{c}\text { No } \\
\text { Pertenece }\end{array}$ & & & Pertenece & $\begin{array}{c}\text { No } \\
\text { Pertenece }\end{array}$ & & \\
\hline EMI & 35,07 & 3,77 & 424,273 & $\star \star \star \star ~$ & 19,4 & 2,46 & 311,74 & 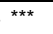 & 15,68 & 1,31 & 515,402 & $2 * \star *$ \\
\hline PREP & 3,24 & 6,07 & 41,145 & 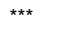 & 2,17 & 3,71 & 31,185 & $* \star \star *$ & 1,07 & 2,36 & 48,656 & $b^{* \star *}$ \\
\hline ASOCAUD & 14,33 & 3,90 & 98,701 & 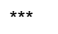 & 8,89 & 2,50 & 95,570 & 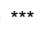 & 5,44 & 1,40 & 83,161 & $1 * \star *$ \\
\hline FIRMAUD & 93,00 & 4,11 & 523,789 & $\star \star \star \star ~$ & 51,3 & 2,64 & 392,26 & 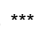 & 41,7 & 1,47 & 619,416 & $b^{* \star *}$ \\
\hline EUR & 5,58 & 3,77 & 15,319 & 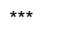 & 3,75 & 2,31 & 25,143 & $* \star \star \star$ & 1,83 & 1,46 & 3,572 & $2 *$ \\
\hline ANGLO & 3,45 & 5,03 & 12,767 & $\star \star \star *$ & 2,29 & 3,10 & 8,653 & *** & 1,16 & 1,92 & 17,243 & $3 * \star \star$ \\
\hline
\end{tabular}

Variables Dependientes: CarPar es el número de cartas emitidas por cada participante en todos los proyectos. CarPar1 es el número de cartas emitidas por cada participante en los proyectos del periodo 2004 a 2010. CarPar2 es el número de cartas emitidas por cada participante en los proyectos del periodo 2011 a 2015. Factores: EMI es una variable dicotómica que toma el valor 2 si es un emisor de normas contables y 1 en otro caso. PREP toma el valor si el participante es preparador de la información. ASOCAUD toma el valor 2 si el participante es una asociación o regulador de auditores. FIRMAUD toma el valor 2 si es una firma internacional de auditoría y 1 en otro caso. EUR toma el valor 2 si el participante procede Europa. ANGLO toma el valor 2 cuando el país de proceedencia pertenece a un sistema jurídico anglosajón.

*** Significativo al 99\%

* Significativo al 90\%

n.s. No significativo

Fuente: Fundación IFRS y elaboración propia. 
Por su parte, los participantes procedentes de países anglosajones también muestran menores niveles de participación que los procedentes de países con sistemas de civil law. Igualmente, los participantes europeos muestran un nivel de implicación ligeramente superior al de otras áreas geográficas.

La Tabla 5 muestra nuestros resultados multivariantes. Hemos desarrollado 3 modelos en los que la variable dependiente se construye con las observaciones de todo el periodo de 2004 a 2015, el modelo 2 con las observaciones del periodo 2004-2010 y el modelo 3 con las del periodo 2011 a 2015.

\section{Tabla 5}

Modelos de regresión con todos los participantes

\begin{tabular}{|c|c|c|c|c|c|c|c|}
\hline \multirow[b]{2}{*}{ Variables } & \multirow{2}{*}{$\begin{array}{c}\text { Signo } \\
\text { previsto }\end{array}$} & $\begin{array}{r}\text { Model } \\
\text { Var. Dep: }\end{array}$ & arPar & \multicolumn{2}{|c|}{$\begin{array}{l}\text { Modelo } 2 \\
\text { Var. Dep: } \\
\text { CarPar1 }\end{array}$} & \multicolumn{2}{|c|}{$\begin{array}{c}\text { Modelo } 3 \\
\text { Var. Dep: CarPar2 }\end{array}$} \\
\hline & & Coef. & sig & Coef. & sig & Coef. & sig \\
\hline Constante & & 2,776 & $\star \star \star *$ & 1,377 & 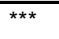 & 1,402 & $\star \star \star ~$ \\
\hline EMI & + & 31,546 & $\star \star \star$ & 17,212 & $\star \star \star$ & 14,333 & $\star \star \star ~$ \\
\hline PREP & - & $-0,191$ & n.s. & $-0,039$ & n.s. & $-0,151$ & n.s. \\
\hline ASOCAUD & + & 10,945 & $\star \star \star *$ & 6,761 & $\star \star \star ~$ & 4,183 & $\star \star \star *$ \\
\hline EUR & & 1,740 & $\star \star \star ~$ & 1,566 & $\star \star \star ~$ & 0,172 & n.s. \\
\hline ANGLO & & 0,076 & n.s. & 0,409 & n.s. & $-0,337$ & n.s. \\
\hline $\mathrm{R}^{2}$ ajustado & & 0,136 & & 0,112 & & 0,149 & \\
\hline$F$ & & 108,205 & $\star \star \star ~$ & 87,118 & $\star \star \star \star$ & 120,540 & $* \star *$ \\
\hline DW & & 1,903 & & 1,926 & & 1,864 & \\
\hline
\end{tabular}

Variables Dependientes: CarPar es el número de cartas emitidas por cada participante en todos los proyectos. CarPar1 es el número de cartas emitidas por cada participante en los proyectos del periodo 2004 a 2010. CarPar2 es el número de cartas emitidas por cada participante en los proyectos del periodo 2011 a 2015. Variables Independientes: EMI es una variable dicotómica que toma el valor 1 si es un emisor de normas contables y 0 en otro caso. PREP toma el valor si el participante es preparador de la información. ASOCAUD toma el valor 1 si el participante es una asociación o regulador de auditores. EUR toma el valor 1 si el participante procede Europa. ANGLO toma el valor 1 cuando el país de proceedencia pertenece a un sistema jurídico anglosajón.

*** Significativo al 99\%

** Significativo al 95\%

n.s. No significativo.

Los FIV se sitúan en todas las variables independientes por debajo de 2 por lo que no se observan problemas de multicolinealidad.

Fuente: Fundación IFRS y elaboración propia.

Los resultados confirman en los tres modelos nuestras hipótesis H1b y H2, observándose una relación significativa y positiva, como preveíamos, entre los emisores de normas contables y las asociaciones de auditores con su nivel de implicación en el due process. El valor de los coeficientes de sendas variables explicativas muestra la mayor implicación de los emisores de normas contables que la de las asociaciones de auditores.

Por su parte, los preparadores muestran un signo negativo, revelando un comportamiento más esporádico que la media de participantes (como ya 
apuntaban los resultados del Análisis de Varianza), si bien no encontramos que el coeficiente sea significativo por lo que tenemos que rechazar nuestra H3.

Las variables de control muestran que la procedencia europea implica un mayor nivel de compromiso que los participantes no europeos, siendo significativa en el modelo 1 y 2; es decir, esta relación comienza a no observase en los últimos años. Por su parte, la tradición jurídica no influye en el nivel de implicación.

Estos resultados confirman que las entidades que por su campo de actuación pueden tener un criterio sobre un amplio abanico de cuestiones son las que presentan un nivel superior de implicación. Los emisores de normas nacionales no pueden dar respuestas individuales a la globalización de la información financiera sino en el seno de mecanismos de cooperación internacional. En esta construcción nacional, el respaldo de los órganos competentes en el ámbito nacional es un input con valor político. Por otra parte, estas entidades se enfrentan a la tarea de evaluar en qué medida una modificación normativa o una nueva norma considera las particularidades institucionales de su jurisdicción y cómo podría afectar a la coordinación con otros marcos normativos de información financiera vigentes en dichos países.

\subsection{Análisis adicionales}

Complementariamente a los resultados anteriores, tratamos de identificar quiénes son las entidades que lideran la participación en el due process del IASB. La Tabla 6 muestra el ranking de las entidades que tienen un compromiso muy elevado con el IASB, participando al menos en el 90\% de los 132 proyectos estudiados. En este grupo se encuentran 5 firmas internacionales (las Big 4 y Grant Thornton), 8 emisores de normas contables nacionales, de los cuales 5 tenían tradición jurídica anglosajona y 3 de modelo legal germánico. Los resultados no presentan a ningún preparador.

Por origen geográfico, Europa aglutina 6 participantes altamente implicados, casi todos ellos emisores de normas contables, incluido el EFRAG. Este resultado muestra cómo la intervención del EFRAG no limita la contribución directa de los participantes más frecuentes. De Asia y Oceanía existen tres participantes altamente comprometidos, mientras que África y Norteamérica están presentes con una institución. Entre estos participantes se encuentran 3 de los 5 miembros del G4+1, faltando el FASB que desarrolla su propio marco y el emisor de normas neozelandés que participa regularmente (79 cartas de 132 proyectos). 


\section{Tabla 6}

Participantes en más del 90\% de los proyectos estudiados entre 2004-2015

(Total = 132 proyectos)

\begin{tabular}{|c|c|c|c|c|c|c|}
\hline Orden & Entidades & Ámbito (País) & $\begin{array}{c}\text { Ámbito } \\
\text { (Continente) }\end{array}$ & Tradición legal $^{1}$ & Colectivo & $\begin{array}{c}\mathrm{N}^{\circ} \text { de } \\
\text { cartas } \\
\text { en todo } \\
\text { el } \\
\text { periodo }\end{array}$ \\
\hline 1 & European Financial Reporting Advisory Group & Europa & Europa & N/A & Emisor de normas & 131 \\
\hline 1 & KPMG & Mundial & Mundial & N/A & Firma internacional & 131 \\
\hline 3 & Deloitte & Mundial & Mundial & N/A & Firma internacional & 130 \\
\hline 3 & South Africa Institute of Chartered Accountants & Sudáfrica & África & Anglosajón & Asociación de auditores & 130 \\
\hline 5 & $\mathrm{PwC}$ & Mundial & Mundial & N/A & Firma internacional & 129 \\
\hline 6 & Korean Accounting Standards Board & Corea del Sur & Asia y Oceanía & Legal germánico & Emisor de normas & 128 \\
\hline 7 & Ernst \& Young & Mundial & Mundial & N/A & Firma internacional & 127 \\
\hline 7 & Malaysian Accounting Standards Board & Malasia & Asia y Oceanía & Anglosajón & Emisor de normas & 127 \\
\hline 9 & Australian Accounting Standards Board & Australia & Asia y Oceanía & Anglosajón & Emisor de normas & 126 \\
\hline 10 & Institute of Chartered Accountants of England \& Wales & Reino Unido & Europa & Anglosajón & Asociación de auditores & 124 \\
\hline 11 & Deutsches Rechnungslegungs Standards Committee & Alemania & Europa & Legal germánico & Emisor de normas & 123 \\
\hline 12 & Financial Reporting Council & Reino Unido & Europa & Anglosajón & Emisor de normas & 122 \\
\hline 13 & Canadian Acounting Standards Board & Canadá & Norteamérica & Anglosajón & Emisor de normas & 121 \\
\hline 13 & Dutch Accounting Standards Board & Holanda & Europa & Legal francés & Emisor de normas & 121 \\
\hline 15 & Grant Thornton & Mundial & Mundial & N/A & Firma internacional & 120 \\
\hline 16 & Association of Chartered Certified Accountants & Reino Unido & Europa & Anglosajón & Asociación de auditores & 119 \\
\hline
\end{tabular}

Se seleccionan las entidades que han en más de un 90\% de los proyectos analizados (Al menos 118 proyectos).

${ }^{1}$ Tradición legal según Laporta et al. (1998).

Fuente: Fundación IFRS y elaboración propia.

Si el criterio de implicación lo ampliamos al $75 \%$ de los proyectos estudiados (Tabla 7), observamos que al perfil reseñado anteriormente se unen dos asociaciones de preparadores, una que agrupa a los directores financieros en Australia (Group of 100 of Finance Directors) y la otra que agrupa a tres asociaciones de empresas francesas (ACTEO, AFEP y MEDEF), también se incorpora el IOSCO cuyo papel en la historia del IASB ha sido determinante para erigirlo en la referencia mundial de la emisión de normas contables.

Tabla 7

Participantes en más del 75\% de los proyectos estudiados entre 2004-2015

(Total = 132 proyectos)

\begin{tabular}{|c|c|c|c|c|c|c|}
\hline Orden & Entidades & Ámbito (País) & $\begin{array}{c}\text { Ámbito } \\
\text { (Continente) }\end{array}$ & Tradición legal $^{1}$ & Colectivo & $\begin{array}{c}\mathrm{N}^{\circ} \text { de } \\
\text { cartas } \\
\text { en todo } \\
\text { el } \\
\text { periodo }\end{array}$ \\
\hline 1 & European Financial Reporting Advisory Group & Europa & Europa & N/A & Emisor de normas & 131 \\
\hline 1 & KPMG & Mundial & Mundial & N/A & Firma internacional & 131 \\
\hline 3 & Deloitte & Mundial & Mundial & $N / A$ & Firma internacional & 130 \\
\hline 3 & South Africa Institute of Chartered Accountants & Sudáfrica & África & Anglosajón & Asociación de auditores & 130 \\
\hline 5 & PwC & Mundial & Mundial & N/A & Firma internacional & 129 \\
\hline 6 & Korean Accounting Standards Board & Corea del Sur & Asia y Oceanía & Legal germánico & Emisor de normas & 128 \\
\hline 7 & Ernst \& Young & Mundial & Mundial & N/A & Firma internacional & 127 \\
\hline 7 & Malaysian Accounting Standards Board & Malasia & Asia y Oceanía & Anglosajón & Emisor de normas & 127 \\
\hline 9 & Australian Accounting Standards Board & Australia & Asia y Oceanía & Anglosajón & Emisor de normas & 126 \\
\hline 10 & Institute of Chartered Accountants of England \& Wales & Reino Unido & Europa & Anglosajón & Asociación de auditores & 124 \\
\hline 11 & Deutsches Rechnungslegungs Standards Committee & Alemania & Europa & Legal germánico & Emisor de normas & 123 \\
\hline
\end{tabular}


Tabla 7 (continuación)

Participantes en más del 75\% de los proyectos estudiados entre 2004-2015

(Total $=132$ proyectos)

\begin{tabular}{|c|c|c|c|c|c|c|}
\hline \multicolumn{2}{|r|}{ Entidades } & \multirow{2}{*}{$\begin{array}{c}\text { Ámbito (País) } \\
\text { Reino Unido }\end{array}$} & \multirow{2}{*}{$\begin{array}{c}\begin{array}{c}\text { Ámbito } \\
\text { (Continente) }\end{array} \\
\text { Europa }\end{array}$} & \multirow{2}{*}{$\begin{array}{c}\text { Tradición legal }^{1} \\
\text { Anglosajón }\end{array}$} & \multirow{2}{*}{$\begin{array}{c}\text { Colectivo } \\
\text { misor de normas }\end{array}$} & \multirow{2}{*}{$\begin{array}{c}\begin{array}{c}N^{\circ} \text { de } \\
\text { cartas } \\
\text { en todo } \\
\text { el } \\
\text { periodo }\end{array} \\
122\end{array}$} \\
\hline 12 & Financial Reporting Council & & & & & \\
\hline 13 & Canadian Acounting Standards Board & Canadá & Norteamérica & Anglosajón & Emisor de normas & 121 \\
\hline 13 & Dutch Accounting Standards Board & Holanda & Europa & Legal francés & Emisor de normas & 121 \\
\hline 15 & Grant Thornton & Mundial & Mundial & N/A & Firma internacional & 120 \\
\hline 16 & Association of Chartered Certified Accountants & Reino Unido & Europa & Anglosajón & Asociación de auditores & 119 \\
\hline 17 & Hong Kong Institute of Certified Public Accountants & Hong Kong & Asia y Oceanía & Anglosajón & Asociación de auditores & 115 \\
\hline 18 & Group of 100 of Finance Directors & Australia & Asia y Oceanía & Anglosajón & Preparadores & 112 \\
\hline 18 & Mazars & Mundial & Mundial & N/A & Firma internacional & 112 \\
\hline 20 & Autorité des Normes Comptables & Francia & Europa & Legal francés & Emisor de normas & 108 \\
\hline 21 & International Organization of Securities Commissions & Mundial & Mundial & N/A & Supervisor de valores & 106 \\
\hline 22 & $\mathrm{BDO}$ & Mundial & Mundial & N/A & Firma internacional & 105 \\
\hline 22 & Japanese Institute of Certified Public Accountants & Japón & Asia y Oceanía & Legal alemán & Asociación de auditores & 105 \\
\hline 24 & Norwegian Accounting Standards Board & Noruega & Europa & Legal escandinavo & Emisor de normas & 103 \\
\hline 25 & ACTEO, AFEP y MEDEF ${ }^{2}$ & Francia & Europa & Legal francés & Preparadores & 102 \\
\hline 26 & Federation de Experts Comptables & Europa & Europa & N/A & Asociación de auditores & 99 \\
\hline
\end{tabular}

Se seleccionan las entidades que han en más de un 75\% de los proyectos analizados (Al menos 99 proyectos)

${ }^{1}$ Tradición legal según Laporta et al. (1998).

${ }^{2}$ ACTEO: Association pour la participation des entreprises françaises à l'harmonisation comptable internationale. AFEP: Association française des entreprises privées. MEDEF: Mouvement des entreprises de France.

Fuente: Fundación IFRS y elaboración propia.

A continuación, dividimos el periodo de observación en dos etapas. Una primera en la que se pueda estudiar la tendencia en los primeros años tras la aplicación en la Unión Europea. Una segunda, en los últimos 5 años, que nos muestre la tendencia. En el periodo 2004-2010, la Tabla 8 muestra que el perfil está equilibrado entre la profesión de auditoría y los emisores de normas contables.

En la Tabla 9, se observa que en los últimos 5 años se incorporan dos entidades procedentes de Asia y Oceanía adicionales y dos entidades de Latinoamérica a las que estaban implicadas en el periodo 2004 a 2010; sin embargo, los colectivos que apoyan al IASB son los auditores y los emisores de normas. La participación recurrente de otros colectivos con información relevante sobre determinados asuntos contables (por ejemplo, las entidades financieras y de seguros sobre instrumentos financieros) es compatible con el marco teórico de colaboración que fundamenta nuestro estudio y podría justificar una participación más selectiva. Esta participación selectiva también podría reflejar comportamientos guiados por el interés propio y la capacidad de poder influir, propio de la teoría económica de la democracia. En este punto, se requieren otros enfoques de investigación que indaguen en los posicionamientos concretos y poder afirmar si la participación ofrece puntos de vista a considerar por el IASB o existe un sesgo motivado por el impacto de la normativa en las cifras contables. 
Tabla 8

Participantes en los proyectos emitidos en el periodo 2004-2010

(Total $=79$ proyectos)

\begin{tabular}{|c|c|c|c|c|c|c|c|}
\hline Orden & Entidades & $\begin{array}{l}\text { Ámbito } \\
\text { (País) }\end{array}$ & $\begin{array}{c}\text { Ámbito } \\
\text { (Continente) }\end{array}$ & Tradición legal $^{1}$ & Colectivo & $\begin{array}{c}\mathrm{N}^{\circ} \\
\text { cartas } \\
\text { antes } \\
\text { de } \\
2011\end{array}$ & $\begin{array}{c}\mathrm{N}^{\circ} \text { de } \\
\text { cartas en } \\
\text { todo el } \\
\text { periodo }\end{array}$ \\
\hline 1 & European Financial Reporting Advisory Group & Europa & Europa & N/A & Emisor de normas & 79 & 131 \\
\hline 1 & KPMG & Mundial & Mundial & N/A & Firma internacional & 79 & 131 \\
\hline 3 & Dutch Accounting Standards Board & Holanda & Europa & Legal francés & Emisor de normas & 78 & 121 \\
\hline 3 & PwC & Mundial & Mundial & N/A & Firma internacional & 78 & 129 \\
\hline 5 & Deloitte & Mundial & Mundial & N/A & Firma internacional & 77 & 130 \\
\hline 5 & Ernst \& Young & Mundial & Mundial & N/A & Firma internacional & 77 & 127 \\
\hline 5 & Korean Accounting Standards Board & Corea del Sur & Asia y Oceanía & Legal germánico & Emisor de normas & 77 & 128 \\
\hline 5 & $\begin{array}{l}\text { South Africa Institute of Chartered } \\
\text { Accountants }\end{array}$ & Sudáfrica & África & Anglosaón & $\begin{array}{l}\text { Asociación de } \\
\text { auditores }\end{array}$ & 77 & 130 \\
\hline 9 & Financial Reporting Council & Reino Unido & Europa & Anglosaón & Emisor de normas & 76 & 122 \\
\hline 9 & Malaysian Accounting Standards Board & Malasia & Asia y Oceanía & Anglosaón & Emisor de normas & 76 & 127 \\
\hline 11 & Australian Accounting Standards Board & Australia & Asia y Oceanía & Anglosaón & Emisor de normas & 75 & 126 \\
\hline 11 & Federation de Experts Comptables & Europe & Europa & N/A & $\begin{array}{l}\text { Asociación de } \\
\text { auditores }\end{array}$ & 75 & 99 \\
\hline 13 & $\begin{array}{l}\text { Association of Chartered Certified } \\
\text { Accountants }\end{array}$ & Reino Unido & Europa & Anglosaón & $\begin{array}{l}\text { Asociación de } \\
\text { auditores }\end{array}$ & 73 & 119 \\
\hline 13 & Autorité des Normes Comptables & Francia & Europa & Legal francés & Emisor de normas & 73 & 108 \\
\hline 13 & $\begin{array}{l}\text { Deutsches Rechnungslegungs Standards } \\
\text { Committee }\end{array}$ & Alemania & Europa & Legal germánico & Emisor de normas & 73 & 123 \\
\hline 13 & $\begin{array}{l}\text { Institute of Chartered Accountants of England } \\
\& \text { Wales }\end{array}$ & Reino Unido & Europa & Anglosaón & $\begin{array}{l}\text { Asociación de } \\
\text { auditores }\end{array}$ & 73 & 124 \\
\hline 17 & Grant Thornton & Mundial & Mundial & & Firma internacional & 72 & 120 \\
\hline 18 & Canadian Acounting Standards Board & Canadá & Norteamérica & Anglosaón & Emisor de normas & 71 & 121 \\
\hline 18 & Institute of Chartered Accountants of Scotland & Reino Unido & Europa & Anglosaón & $\begin{array}{l}\text { Asociación de } \\
\text { auditores }\end{array}$ & 71 & 90 \\
\hline
\end{tabular}

Se seleccionan las entidades que han en más de un 90\% de los proyectos analizados (Al menos 71 proyectos).

${ }^{1}$ Tradición legal según Laporta et al. (1998).

Fuente: Fundación IFRS y elaboración propia.

Tabla 9

Participantes en los proyectos emitidos en el periodo 2011-2015 (Total $=53$ proyectos)

\begin{tabular}{|c|c|c|c|c|c|c|c|}
\hline Orden & Entidades & $\begin{array}{l}\text { Ámbito } \\
\text { (País) }\end{array}$ & $\begin{array}{c}\text { Ámbito } \\
\text { (Continente) }\end{array}$ & Tradición legal $^{1}$ & Colectivo & $\begin{array}{c}\mathrm{N}^{\circ} \\
\text { cartas } \\
2011- \\
2015\end{array}$ & $\begin{array}{c}\mathrm{N}^{\circ} \mathrm{de} \\
\text { cartas en } \\
\text { todo el } \\
\text { periodo }\end{array}$ \\
\hline 1 & Deloitte & Mundial & Mundial & N/A & Firma internacional & 53 & 130 \\
\hline 1 & $\begin{array}{l}\text { South Africa Institute of Chartered } \\
\text { Accountants }\end{array}$ & Sudáfrica & África & Anglosajón & $\begin{array}{l}\text { Asociación de } \\
\text { auditores }\end{array}$ & 53 & 130 \\
\hline 3 & European Financial Reporting Advisory Group & Europa & Europa & N/A & Emisor de normas & 52 & 131 \\
\hline 3 & $\begin{array}{l}\text { Hong Kong Institute of Certified Public } \\
\text { Accountants }\end{array}$ & Hong Kong & Asia y Oceanía & Anglosajón & $\begin{array}{l}\text { Asociación de } \\
\text { auditores }\end{array}$ & 52 & 115 \\
\hline 3 & KPMG & Mundial & Mundial & N/A & Firma internacional & 52 & 131 \\
\hline 6 & Australian Accounting Standards Board & Australia & Asia y Oceanía & Anglosajón & Emisor de normas & 51 & 126 \\
\hline 6 & $\begin{array}{l}\text { Institute of Chartered Accountants of England } \\
\& \text { Wales }\end{array}$ & Reino Unido & Europa & Anglosajón & $\begin{array}{l}\text { Asociación de } \\
\text { auditores }\end{array}$ & 51 & 124 \\
\hline 6 & Korean Accounting Standards Board & Corea del Sur & r Asia y Oceanía & Legal germánico & Emisor de normas & 51 & 128 \\
\hline 6 & Malaysian Accounting Standards Board & Malasia & Asia y Oceanía & Anglosajón & Emisor de normas & 51 & 127 \\
\hline
\end{tabular}


Tabla 9 (continuación)

Participantes en los proyectos emitidos en el periodo 2011-2015

(Total $=53$ proyectos)

\begin{tabular}{|c|c|c|c|c|c|c|c|}
\hline Orden & Entidades & $\begin{array}{l}\text { Ámbito } \\
\text { (País) }\end{array}$ & $\begin{array}{c}\text { Ámbito } \\
\text { (Continente) }\end{array}$ & Tradición legal $^{1}$ & Colectivo & $\begin{array}{c}\mathrm{N}^{\circ} \\
\text { cartas } \\
2011- \\
2015\end{array}$ & $\begin{array}{c}\mathrm{N}^{\circ} \mathrm{de} \\
\text { s cartas en } \\
\text { todo el } \\
\text { periodo }\end{array}$ \\
\hline 6 & PwC & Mundial & Mundial & $\mathrm{N} / \mathrm{A}$ & Firma internacional & 51 & 129 \\
\hline 11 & $\begin{array}{l}\text { Canadian Acounting Standards Board } \\
\text { Consejo Mexicano para la Investigación y }\end{array}$ & Canadá & Norteamérica & Anglosajón & Emisor de normas & 50 & 121 \\
\hline 11 & $\begin{array}{l}\text { Desarrollo de las Normas de Información } \\
\text { Financiera }\end{array}$ & México & Latinoamérica & Legal francés & Emisor de normas & 50 & 98 \\
\hline 11 & Ernst \& Young & Mundial & Mundial & N/A & Firma internacional & 50 & 127 \\
\hline 11 & $\begin{array}{l}\text { Deutsches Rechnungslegungs Standards } \\
\text { Committee }\end{array}$ & Alemania & Europa & Legal germánico & Emisor de normas & 50 & 123 \\
\hline 11 & Singapore Accounting Standards Committee & Singapur & Asia y Oceanía & Anglosajón & Emisor de normas & 50 & 97 \\
\hline 16 & Comité Pronunciamentos Contabeis & Brasil & Latinoamérica & Legal francés & Emisor de normas & 48 & 65 \\
\hline 16 & Grant Thornton & Mundial & Mundial & N/A & Firma internacional & 48 & 120 \\
\hline 16 & Mazars & Mundial & Mundial & N/A & Firma internacional & 48 & 112 \\
\hline 16 & Norwegian Accounting Standards Board & Noruega & Europa & $\begin{array}{l}\text { Legal } \\
\text { escandinavo }\end{array}$ & Emisor de normas & 48 & 103 \\
\hline 20 & European Securities and Markets Authorities & Europa & Europa & N/A & $\begin{array}{l}\text { Supervisor del } \\
\text { mercado }\end{array}$ & 47 & 61 \\
\hline
\end{tabular}

Se seleccionan las entidades que han en más de un 90\% de los proyectos analizados (Al menos 47 proyectos).

${ }^{1}$ Tradición legal según Laporta et al. (1998).

Fuente: Fundación IFRS y elaboración propia.

Las instituciones con niveles elevados de colaboración representan a amplios colectivos, incluidas las firmas internacionales de auditoría que agrupan a los despachos nacionales de dichas firmas. La necesidad de recursos para mantener un permanente feedback con el IASB es un esfuerzo notable que, unido a otras contribuciones, pone de manifiesto cómo la solución de la armonización contable internacional requiere un esfuerzo colectivo importante.

La Tabla 10 muestra cómo los emisores de normas asiáticos y europeos son los más comprometidos con la labor del IASB, lo cual es un indicio del respaldo político de los gobiernos de esas regiones a la armonización contable internacional liderada por el IASB. Asimismo, observamos cómo algunos emisores (el Consejo Mexicano para la Investigación y Desarrollo de las Normas de Información Financiera, el Singapore Accounting Standards Committee, el Comitê de Pronunciamentos Contábeis, el China Accounting Standards Committee y el Instituto de Contabilidad y Auditoría de Cuentas) tienen una participación superior en los últimos 5 años, frente a los 7 años anteriores. Merece una mención el papel de la Asian Oceanian Standards Setters Group que con 45 cartas enviadas (38 en los últimos 5 años) y el Group of Latin American Standard Setters con 43 envíos, todos ellos en los últimos 5 años, han surgido con el European Financial Reporting Advisory Group como participantes que representan los intereses de los emisores de normas contables nacionales y un cauce eficiente para participar proactivamente en la formulación de la normativa que posteriormente está vigente en su jurisdicción. 
Tabla 10

20 primeros emisores de normas contables por número de cartas emitidas en el periodo 2004-2015

(Total = 132 proyectos)

\begin{tabular}{|c|c|c|c|c|c|c|c|}
\hline Orden & Entidades & Ámbito (País) & $\begin{array}{c}\text { Ámbito } \\
\text { (Continente) }\end{array}$ & Tradición legal $^{1}$ & $\begin{array}{c}N^{\circ} \text { de } \\
\text { cartas } \\
\text { en todo } \\
\text { el } \\
\text { periodo }\end{array}$ & $\begin{array}{c}N^{\circ} \text { cartas } \\
\text { antes de } \\
2011 \\
(n=79)\end{array}$ & $\begin{array}{c}\mathrm{N}^{\circ} \text { cartas } \\
\text { en el } \\
\text { periodo } \\
2011-2015 \\
(n=53)\end{array}$ \\
\hline 1 & European Financial Reporting Advisory Group & Europa & Europa & N/A & 131 & 79 & 52 \\
\hline 2 & Korean Accounting Standards Board & Corea del Sur & Asia y Oceanía & Legal escandinavo & 128 & 77 & 51 \\
\hline 3 & Malaysian Accounting Standards Board & Malasia & Asia y Oceanía & Anglosajón & 127 & 76 & 51 \\
\hline 4 & $\begin{array}{l}\text { Australian Accounting Standards Board } \\
\text { Deutsches Rechnungslegungs Standards }\end{array}$ & Australia & Asia y Oceanía & Anglosajón & 126 & 75 & 51 \\
\hline 5 & Committee & Alemania & Europa & Legal germánico & 123 & 73 & 50 \\
\hline 6 & Financial Reporting Council & Reino Unido & Europa & Anglosajón & 122 & 76 & 46 \\
\hline 7 & Canadian Acounting Standards Board & Canadá & Norteamérica & Anglosajón & 121 & 71 & 50 \\
\hline 7 & Dutch Accounting Standards Board & Holanda & Europa & Legal francés & 121 & 78 & 43 \\
\hline 9 & Autorité des Normes Comptables & Francia & Europa & Legal francés & 108 & 73 & 35 \\
\hline 10 & Norwegian Accounting Standards Board & Noruega & Europa & Legal escandinavo & 103 & 55 & 48 \\
\hline 11 & $\begin{array}{l}\text { Consejo Mexicano para la Investigación y } \\
\text { Desarrollo de las Normas de Información } \\
\text { Financiera }\end{array}$ & México & Latinoamérica & Legal francés & 98 & 48 & 50 \\
\hline 12 & Singapore Accounting Standards Committee & Singapur & Asia y Oceanía & Anglosajón & 97 & 47 & 50 \\
\hline 13 & Swedish Financial Reporting Board ${ }^{2}$ & Suecia & Europa & Legal escandinavo & 91 & 58 & 33 \\
\hline 14 & Accounting Standards Board of Japan & Japón & Asia y Oceanía & Legal germánico & 88 & 55 & 33 \\
\hline 14 & Organismo Italiano di Contabilità & Italia & Europa & Legal francés & 88 & 56 & 32 \\
\hline 16 & $\begin{array}{l}\text { New Zealand Accounting Standards Board } \\
\text { (External Reporting Board) }\end{array}$ & Nueva Zelanda & Asia y Oceanía & Anglosajón & 79 & 52 & 27 \\
\hline 17 & Comitê de Pronunciamentos Contábeis & Brasil & Latinoamérica & Legal francés & 65 & 17 & 48 \\
\hline 18 & China Accounting Standards Committee & China & Asia y Oceanía & Legal germánico & 62 & 25 & 37 \\
\hline 19 & $\begin{array}{l}\text { Austrian Financial Reporting and Auditing } \\
\text { Committee }\end{array}$ & Austria & Europa & Legal germánico & 61 & 38 & 23 \\
\hline 20 & Instituto de Contabilidad y Auditoría de Cuentas & España & Europa & Legal francés & 59 & 23 & 36 \\
\hline
\end{tabular}

Fuente: Fundación IFRS y elaboración propia.

Los emisores de normas contables pertenecen a diferentes tradiciones jurídicas, por lo que en este punto se puede considerar aceptable el nivel de diversidad.

Las asociaciones de auditores que participan son fundamentalmente de origen anglosajón. Este patrón de comportamiento es compatible con la evidencia que concluía que la familiaridad con el estilo de regulación facilitaba mayores niveles de participación (Dobler y Knosper, 2016). La procedencia geográfica está distribuida de manera equilibrada entre Asia y Europa, como en el caso de los emisores. 
Tabla 11

20 primeras asociaciones o reguladores de auditores, por número de cartas emitidas en el periodo 2004-2015

(Total $=132$ proyectos)

\begin{tabular}{|c|c|c|c|c|c|c|c|}
\hline Orden & Entidades & Ámbito (País) & $\begin{array}{c}\text { Ámbito } \\
\text { (Continente) }\end{array}$ & Tradición legal $^{1}$ & $\begin{array}{c}\mathrm{N}^{\circ} \text { de } \\
\text { cartas } \\
\text { en todo } \\
\text { el } \\
\text { periodo }\end{array}$ & $\begin{array}{c}N^{\circ} \text { cartas } \\
\text { antes de } \\
2011 \\
(n=79)\end{array}$ & $\begin{array}{c}\mathrm{N}^{\circ} \text { cartas } \\
\text { en el } \\
\text { periodo } \\
2011-2015 \\
(n=53)\end{array}$ \\
\hline 1 & $\begin{array}{l}\text { Association of Chartered Certified Accountants } \\
\text { Hong Kong Institute of Certified Public }\end{array}$ & Reino Unido & Europa & Anglosajón & 119 & 73 & 46 \\
\hline 2 & $\begin{array}{l}\text { Accountants } \\
\text { Japanese Institute of Certified Public }\end{array}$ & Hong Kong & Asia y Oceanía & Anglosajón & 115 & 63 & 52 \\
\hline 3 & Accountants & Japón & Asia y Oceanía & Legal germánico & 105 & 59 & 46 \\
\hline 4 & Federation de Experts Comptables & Europa & Europa & N/A & 99 & 75 & 24 \\
\hline 5 & Institute for Accounting Profession in Sweden & Suecia & Europa & Legal escandinavo & 98 & 67 & 31 \\
\hline 6 & Institute of Chartered Accountants of Ireland & Irlanda & Europa & Anglosajón & 97 & 69 & 28 \\
\hline 7 & Institut der Wirtschatsprüfer & Alemania & Europa & Legal germánico & 91 & 67 & 24 \\
\hline 8 & Institute of Chartered Accountants of Scotland & Reino Unido & Europa & Anglosajón & 90 & 71 & 19 \\
\hline 9 & $\begin{array}{l}\text { Institute of Chartered Accountants of Pakistan } \\
\text { Institute of Certified Public Accountants of }\end{array}$ & Pakistán & Asia y Oceanía & Anglosajón & 78 & 52 & 26 \\
\hline 10 & Singapore & Singapur & Asia y Oceanía & Anglosajón & 68 & 33 & 35 \\
\hline 10 & Zambia Institute of Chartered Accountants & Zambia & África & Anglosajón & 68 & 31 & 37 \\
\hline 12 & $\begin{array}{l}\text { Institute of Chartered Accountants of India } \\
\text { Institute of Certified of Public Accountants of }\end{array}$ & India & Asia y Oceanía & Anglosajón & 52 & 27 & 25 \\
\hline 12 & Kenya & Kenia & África & Anglosajón & 52 & 18 & 34 \\
\hline 14 & Certified Public Accountants of Australia & Australia & Asia y Oceanía & Anglosajón & 51 & 29 & 22 \\
\hline 15 & Federation de Experts Comptables & Dinamarca & Europa & Legal escandinavo & 43 & 40 & 3 \\
\hline 16 & $\begin{array}{l}\text { London Society of Chartered Accountants } \\
\text { Institute of Certified of Public Accountants of }\end{array}$ & Reino Unido & Europa & Anglosajón & 36 & 35 & 1 \\
\hline 17 & Ireland & Irlanda & Europa & Anglosajón & 34 & 18 & 16 \\
\hline 17 & National Institute of Accountants in Australia & Australia & Asia y Oceanía & Anglosajón & 34 & 27 & 7 \\
\hline 19 & Certified General Accountants Comptables & Canadá & Norteamérica & Anglosajón & 29 & 22 & 7 \\
\hline 20 & Institute of Chartered Accountants in Australia & Australia & Asia y Oceanía & Anglosajón & 29 & 19 & 10 \\
\hline
\end{tabular}

${ }^{1}$ Tradición legal según Laporta et al. (1998).

Fuente: Fundación IFRS y elaboración propia.

Tabla 12

Firmas internacionales de auditoría, por número de cartas emitidas en el periodo 20042015

(Total $=132$ proyectos)

\begin{tabular}{|clccc|}
\hline Orden & Entidades & $\begin{array}{c}\mathbf{N}^{\circ} \text { de cartas en todo el } \\
\text { periodo }\end{array}$ & $\begin{array}{c}\mathbf{N}^{\circ} \text { cartas antes de 2011 } \\
(\mathbf{n}=\mathbf{7 9})\end{array}$ & $\begin{array}{c}\mathbf{N}^{\circ} \text { cartas en el periodo } \\
\mathbf{2 0 1 1 - 2 0 1 5}(\mathbf{n}=53)\end{array}$ \\
\hline 1 & KPMG & 131 & 79 & 52 \\
2 & Deloitte & 130 & 77 & 53 \\
3 & PwC & 129 & 78 & 51 \\
4 & Ernst \& Young & 127 & 77 & 50 \\
5 & Grant Thornton & 120 & 72 & 48 \\
6 & Mazars & 112 & 64 & 48 \\
7 & BDO & 105 & 60 & 45 \\
8 & RSM & 45 & 4 & 41 \\
9 & Moore Stephens & 16 & 0 & 16 \\
10 & Crowe Horwath & 15 & 2 & 13 \\
\hline
\end{tabular}

${ }^{1}$ Tradición legal según Laporta et al. (1998).

Fuente: Fundación IFRS y elaboración propia.

Las firmas internacionales de auditoría están apoyando este proceso de armonización contable internacional desde los orígenes del IASC y atienden 
puntualmente la práctica totalidad de consultas, incluso las que implican correcciones menores. Asimismo, observamos la irrupción de tres redes de auditoría internacional como son RSM, Moore Stephens y Crowe Horwarth.

Los preparadores no financieros y de seguros muestran un menor nivel de respuesta que los colectivos precedentes, en primer lugar, porque el conocimiento a comunicar se limita a las problemáticas contables con las que están familiarizados y, en segundo lugar, porque su motivación a participar podría explicarse por otros marcos teóricos centrados en el propio interés, como el formulado por Sutton (1984) o por la teoría positiva de la contabilidad. En este colectivo, la procedencia de las empresas, o asociaciones de empresas, es fundamentalmente europea. La mitad de estos participantes son asociaciones y la otra mitad, empresas de gran dimensión, de sectores diversos como petróleo y gas, alimentación, energía, automóviles, entre otros.

Tabla 13

20 primeras empresas y asociaciones empresariales, no financieras ni de seguros, por número de cartas emitidas en el periodo 2004-2015

(Total $=132$ proyectos)

\begin{tabular}{|c|c|c|c|c|c|c|c|}
\hline Orden & Entidades & Ámbito (País) & $\begin{array}{c}\text { Ámbito } \\
\text { (Continente) }\end{array}$ & Tradición legal $^{1}$ & $\begin{array}{c}\mathrm{N}^{\circ} \text { de } \\
\text { cartas } \\
\text { en todo } \\
\text { el } \\
\text { periodo }\end{array}$ & $\begin{array}{c}N^{\circ} \text { cartas } \\
\text { antes de } \\
2011 \\
(n=79)\end{array}$ & $\begin{array}{c}\mathrm{N}^{\circ} \text { cartas } \\
\text { en el } \\
\text { periodo } \\
2011-2015 \\
(n=53)\end{array}$ \\
\hline 1 & Group of 100 of finance directors & Australia & Asia y Oceanía & Anglosajón & 112 & 69 & 43 \\
\hline 2 & ACTEO-AFEP-MEDEF & Francia & Europa & Legal francés & 102 & 63 & 39 \\
\hline 3 & BusinessEurope (UNICE) & Bélgica & Europa & Legal francés & 87 & 52 & 35 \\
\hline 4 & Swiss Holdings & Suiza & Europa & Legal germánico & 75 & 43 & 32 \\
\hline 5 & Swedish Entrerprise Accounting Association & Suecia & Europa & Legal escandinavo & 69 & 37 & 32 \\
\hline 6 & Hoffman La Roche & Suiza & Europa & Legal germánico & 64 & 51 & 13 \\
\hline 7 & Finacial executives International (Canadá) & Canadá & Norteamérica & Anglosajón & 63 & 36 & 27 \\
\hline 8 & Nestlé & Suiza & Europa & Legal germánico & 59 & 46 & 13 \\
\hline 9 & Chartered Institute of Management Accountants & Mundial & Mundial & N/A & 54 & 50 & 4 \\
\hline 10 & Hundred Group of Finance Directors & Reino Unido & Europa & Anglosajón & 48 & 37 & 11 \\
\hline 11 & Shell & Reino Unido & Europa & Anglosajón & 44 & 32 & 12 \\
\hline 12 & Hydro Quebec & Canadá & Norteamérica & Anglosajón & 43 & 19 & 24 \\
\hline 12 & Quoted Companies Alliance & Reino Unido & Europa & Anglosajón & 43 & 26 & 17 \\
\hline 14 & Confedereation of British Industry & Reino Unido & Europa & Anglosajón & 42 & 36 & 6 \\
\hline 15 & BP plc & Reino Unido & Europa & Anglosajón & 40 & 21 & 19 \\
\hline 15 & Syngenta & Switzerland & Europa & Legal germánico & 40 & 28 & 12 \\
\hline 15 & Telstra & Australia & Asia y Oceanía & Anglosajón & 40 & 34 & 6 \\
\hline 18 & Repsol & España & Europa & Legal francés & 39 & 6 & 33 \\
\hline \multirow{2}{*}{$\begin{array}{l}19 \\
20\end{array}$} & \multirow{2}{*}{$\begin{array}{l}\text { Volkswagen } \\
\text { International Swaps and Derivatives } \\
\text { Association }\end{array}$} & Alemania & Europa & Legal germánico & 37 & 22 & 15 \\
\hline & & Mundial & Mundial & N/A & 36 & 26 & 10 \\
\hline
\end{tabular}

${ }^{1}$ Tradición legal según Laporta et al. (1998).

${ }^{2}$ ACTEO: Association pour la participation des entreprises françaises à l'harmonisation comptable internationale. AFEP: Association française des entreprises privées. MEDEF: Mouvement des entreprises de France.

Fuente: Fundación IFRS y elaboración propia.

Los preparadores financieros y de seguros participan más selectivamente, pues su negocio se centra en determinadas áreas de los estados financieros. Así, los proyectos sobre instrumentos financieros han generado un gran interés en 
este colectivo. El sector bancario participa más activamente que el de seguros y, como en el caso de los preparadores no financieros, la mitad son asociaciones. El origen geográfico dominante de las entidades que presentan un mayor nivel de implicación es Europa.

Tabla 14

20 primeras entidades financieras y de seguros, por número de cartas emitidas en el periodo 2004-2015

(Total $=132$ proyectos)

\begin{tabular}{|c|c|c|c|c|c|c|c|}
\hline Orden & Entidades & Ámbito (País) & $\begin{array}{c}\text { Ámbito } \\
\text { (Continente) }\end{array}$ & Tradición legal $^{1}$ & $\begin{array}{c}\mathrm{N}^{\circ} \text { de } \\
\text { cartas } \\
\text { en todo } \\
\text { el } \\
\text { periodo }\end{array}$ & $\begin{array}{c}N^{\circ} \text { cartas } \\
\text { antes de } \\
2011 \\
(n=79)\end{array}$ & $\begin{array}{c}N^{\circ} \text { cartas } \\
\text { en el } \\
\text { periodo } \\
2011-2015 \\
(n=53)\end{array}$ \\
\hline 1 & UBS & Suiza & Europa & Legal germánico & Bancos & 66 & 52 \\
\hline 2 & Canadian Bankers Association & Canadá & Norteamérica & Anglosajón & Bancos & 57 & 35 \\
\hline 3 & British Bankers' Association & Reino Unido & Europa & Anglosajón & Bancos & 55 & 46 \\
\hline 3 & HSBC & Reino Unido & Europa & Anglosajón & Bancos & 55 & 42 \\
\hline 5 & Life Insurance Association of Japan & Japón & Asia y Oceanía & Legal germánico & Seguros & 50 & 33 \\
\hline 6 & Association of British Insurers & Reino Unido & Europa & Anglosajón & Seguros & 47 & 35 \\
\hline 6 & ANZ & Australia & Asia y Oceanía & Anglosajón & Bancos & 47 & 29 \\
\hline 6 & Deutsche Bank & Alemania & Europa & Legal germánico & Bancos & 47 & 31 \\
\hline 9 & BNP Paribas & Francia & Europa & Legal francés & Bancos & 46 & 35 \\
\hline 10 & Allianz & Alemania & Europa & Legal germánico & Seguros & 45 & 29 \\
\hline 11 & Federation Bancaire Française & Francia & Europa & Legal francés & Bancos & 42 & 28 \\
\hline 11 & Hong Kong Association of Banks & Hong Kong & Asia y Oceanía & Anglosajón & Bancos & 42 & 10 \\
\hline 13 & Credit Suisse Group & Suiza & Europa & Legal germánico & Bancos & 39 & 28 \\
\hline 14 & London Investment Banking Association & Reino Unido & Europa & Anglosajón & Bancos & 35 & 35 \\
\hline 15 & Barclays & Reino Unido & Europa & Anglosajón & Bancos & 33 & 20 \\
\hline 15 & European Banking Federation & Europa & Europa & N/A & Bancos & 33 & 23 \\
\hline 15 & German Insurance Association & Alemania & Europa & Legal germánico & Seguros & 33 & 12 \\
\hline 18 & Bundesverband Deutscher Banken & Alemania & Europa & Legal germánico & Bancos & 31 & 28 \\
\hline 18 & European Association of Cooperative Banks & Europa & Europa & Legal germánico & Bancos & 31 & 20 \\
\hline 20 & National Audtralia Bank & Australia & Asia y Oceanía & Anglosajón & Bancos & 30 & 21 \\
\hline
\end{tabular}

${ }^{1}$ Tradición legal según Laporta et al. (1998).

Fuente: Fundación IFRS y elaboración propia.

\section{CONCLUSIONES}

Los procesos de consulta abierta son un mecanismo por el cual las organizaciones privadas internacionales tratan de buscar la legitimidad de sus actuaciones. Esta legitimidad en el input se considera crítica y ha dado lugar a la puesta en marcha de acciones tendentes a dinamizarla. La legitimidad en el input se mide por el número de cartas recibidas, pero también por la calidad de esas cartas, medida ésta por la diversidad geográfica y de colectivos participantes.

Nuestro trabajo trata de introducir otra dimensión de la calidad del input, basado en el modelo analítico de Königsgruber (2013), que es la capacidad del participante por aportar información relevante. La competencia técnica de las entidades que participan, unida a la diversidad que aporta su origen geográfico y su papel en la cadena de información, permiten enriquecer el proceso deliberativo que aborda el IASB. Nuestros resultados han puesto de manifiesto que la 
participación más recurrente se da cita principalmente en asociaciones; este mecanismo permite compartir los costes vinculados a la participación. Estas entidades son las referencias en conocimiento experto en el ámbito de la emisión de normas contables nacionales y en el ámbito de la práctica de auditoría.

Los emisores nacionales de normas contables comienzan a asumir su papel proactivo en la cadena de emisión de normas que posteriormente afectará de manera directa a las empresas que aplican las normas internacionales de contabilidad en el ámbito jurisdiccional y, de manera indirecta, por el efecto que los cambios en la normativa contable internacional pueden aconsejar en la normativa contable nacional. Para el IASB, la participación de los emisores de normas nacionales es un input de especial calidad técnica y de gran valor político, pues normalmente estas entidades son supervisadas y rinden cuentas a las autoridades elegidas por los ciudadanos.

Metodológicamente, nuestro trabajo incorpora la dimensión individual en los estudios multi-periodo y multi-proyecto en el análisis del lobbying. Esto lo efectuamos tomando como unidad de muestreo los participantes. A excepción del trabajo de Jorissen et al. (2012), donde se efectúa este análisis para los preparadores de la información financiera en 22 proyectos, no conocemos otro trabajo que asuma un enfoque similar.

El trabajo está sujeto a limitaciones de varios tipos. No ha sido posible analizar el $100 \%$ de proyectos abiertos a consulta en el periodo 2004-2015, al no haber podido obtener las cartas de comentario de dos proyectos. Sin embargo, se trata de dos proyectos que implican una modificación sobre fechas de aplicación y el segundo un proyecto sobre la revisión de la constitución. Una segunda debilidad es el tratamiento de la participación conjunta. Nuestra decisión fue considerar estas asociaciones temporales para enviar una carta como si de un participante se tratase, sin embargo, estas formas de participación colectivas son una vía alternativa a la carta individual. De haber optado por considerarlas como intervenciones de cada uno de sus miembros nuestros indicadores de cartas enviadas podrían estar ligeramente infravaloradas en algunos participantes.

\section{REFERENCIAS BIBLIOGRÁFICAS}

BAMBER, M. y MCMEEKING, K. (2016). "An examination of international accounting standard-setting due process and the implications for legitimacy". The British Accounting Review, 48(1), pp. 59-73.

BURLAUD, A. y COLASE, B. (2011). "International Accounting Standardisation: Is Politics Back?" Accounting in Europe, 8(1), pp. 23-47. 
CAMFFERMAN, K., y ZEFF, S. A. (2007). Financial reporting and global capital markets: A history of the international accounting standards committee, 1973-2000. Oxford: Oxford University Press.

CAMFFERMAN, K. y ZEFF, S. A. (2017). "The Challenge of Setting Standards for a Worldwide Constituency: Research Implications from the IASB's Early History". European Accounting Review, Vol. 0, Iss. 0

CHIRCOP, J. PARASKEVI, V. K. (2015). "Why did preparers lobby to the IASB's pension accounting proposals?". Accounting Forum, 39(4), pp. 268-280.

DOBLER, M. y KNOSPE, O. (2016a). "Attributes of lobbying towards the IASB: Participation, content, and success". Working paper. Disponible en [Consultado 20/02/2018]: https://business.illinois.edu/accountancy/wp-content/uploads/sites/12/ 2016/01/1615-Revision-1.pdf

DOBLER, M. y KNOSPE, O. (2016b). "Constituents' formal participation in the IASB's Due Process: New Insights into the Impact of Country and Due Process Document Characteristics". Journal of Governance and Regulation, 5(3), pp. 50-66.

DUROCHER, S., FORTIN, A. y CÔTE, L. (2007). "Users' participation in the accounting standard-setting process: a theory-building study". Accounting, Organizations and Society, 32(1-2), pp. 33-63.

DUROCHER, S. y FORTIN, A. (2011). "Practitioners' participation in the accounting standard-setting process". Accounting and Business Research, 41(1), pp. 29-50.

EISENSCHMIDT, K. y KRASODOMSKA, J. (2017). "Insights into the IASB due process: the influence of country characteristics on constituents' formal participation". Zeszyty Teoretyczne Rachunkowosc, 95(151), pp.117-145.

GEORGIOU, G. (2010). "The IASB standard-setting process: Participation and perceptions of financial statement users". The British Accounting Review, 42(2), pp. 103-118

GINER, B. y ARCE, M. (2012). "Lobbying on Accounting Standards: Evidence from IFRS 2 on Share-Based Payments". European Accounting Review, 21(4), pp. 655-691.

GOEDL, P. A. (2012). "Defining the IASB's governance network: a social network analysis". International Journal of Critical Accounting, 4(1), pp. 30-53.

INTERNATIONAL FEDERATION OF ACCOUNTANTS (IFAC), (2008). Financial Reporting Supply Chain. Current Perspectives and Directions. Disponible en [Consultado 20/02/2018]: http://www.ifac.org/sites/default/files/publications/files/ financial-reportingsupply.pdf.

JORISSEN, A., LYBAERT, N., \& VAN DE POEL, K., (2006). "Lobbying towards a global standard setter-do national characteristics matter?: an analysis of the comment letters written to the IASB". En: Gregoriou, G.N. \& Gaber, M., eds. International accounting: standards, regulations, and financial reporting, Oxford: Elsevier, 1-39.

JORISSEN, A., LYBAERT, N., ORENS, R. y VAN DER TAS, L. (2012). "Formal Participation in the lasb's due Process of Standard Setting: A Multi-issue/Multi-period Analysis". European Accounting Review 21(4), pp. 693-729

JORISSEN A., LYBAERT, N., ORENS, R. y VAN DER TAS, L. (2013). "A geographic analysis of constituents' formal participation in the process of international accounting standard setting: Do we have a level playing field?" Journal of Accounting and Public Policy, 32(4), pp. 237-270.

JORISSEN, A., LYBAERT, N., ORENS, R. y VAN DER TAS, L. (2014). "Constituents' Participation in the IASC/IASB's due Process of International Accounting Standard 
Setting: A Longitudinal Analysis". Accounting and Regulation, Ed. Springer. New York, pp. $79-110$

KÖNIGSGRUBER, R. (2014). "Expertise-based lobbying and accounting regulation". Journal of Management \& Governance, 17(4), pp. 1009-1025.

KOSI, U. y REITHER, A. (2014). "Determinants of Corporate Participation in the IFRS 4 (Insurance Contracts) Replacement Process" Accounting in Europe, 11(1), pp. 89-112.

LA PORTA, R., LOPEZ-DE-SILANES, F., SHLEIFER, A., y VISHNY, R. W. (1998). "Law and finance". Journal of Political Economy, 106(6), pp. 1113-1155.

LARSON, R. K. (2007). "Constituent Participation and the IASB's International Financial Reporting Interpretations Committee". Accounting in Europe, 4(2), pp. 207-254.

LARSON, R. K. y HERTZ, P. J. (2013). "A Multi-Issue/Multi-Period Analysis of the Geographic Diversity of IASB Comment Letter Participation". Accounting in Europe, 10(1), pp.99-151.

MACARTHUR, J. B. (2012). "Some Implications of Auditor and Client Lobbying Activities: A Comparative Analysis". Accounting and Business Research, 19(73), pp. 56-64.

MELLADO, L. y PARTE, L. (2017). "Determinants of corporate lobbying intensity in the lease standard-setting process". Spanish Accounting Review, 20(2), pp. 131-142.

MILLER, P. B. W., REDDING, R. J. y BAHNSON, P. (1996). The FASB: The People, the Process, and the Politics. 3th ed. Boston, MA: Irwin/McGraw-Hill

MORLEY, J. (2015). "Internal lobbying at the IASB". Journal of Accounting and Public Policy, 35(3), pp. 224-255.

NÖLKE A. (2015). "Rising Powers and Transnational Private Governance: The International Accounting Standards Board". In: Lesage D., Van de Graaf T. (eds) Rising Powers and Multilateral Institutions. International Political Economy Series. Palgrave Macmillan, London, pp. 96-116.

ORENS, R, JORISSEN, A., LYBAERT, N y VAN DER TAS, L. (2011). "Corporate Lobbying in Private Accounting Standard Setting: Does the IASB have to Reckon with National Differences?". Accounting in Europe, 8(2), pp. 211-234.

PELGER, C. y SPIEß, N. (2017). "On the IASB's construction of legitimacy - the case of the agenda consultation project". Accounting and Business Research, 47(1), pp. 64-90.

PROCHÁZKA, D. (2015). "Lobbying on the IASB Standards: An Analysis of the Lobbyists' Behaviour Over Period 2006-2014". Copernican Journal of Finance \& Accounting, 4(2), pp. $129-143$

RICHARDSON, A. J. y EBERLYN, B. (2011). "Legitimating Transnational Standard Setting: The Case of the International Accounting Standards Board" Journal of Business Ethics, 98, pp. 217-245.

SUTTON, T. G. (1984). "Lobbying of accounting standard setting bodies in the UK and the USA: a Downsian analysis" Accounting, Organizations and Society, 9(1), pp. 81-95.

TANDY, P. R., WILBURN, N. L. (1992). "Constituent Participation in Standard-Setting: The FASB's First 100 Statements". Accounting Horizons, 6(2), pp. 47-58.

WINGARD, C., BOSMAN, J., BRIGHT, A. (2016). "The legitimacy of IFRS: An assessment of the influences on the due process of standard-setting". Meditari Accountancy Research, Vol. 24(1), pp.134-156.

ZIMMERMANN J., WERNER J.R. (2013). "Explaining the Evolution of a New Accounting Framework. In: Regulating Capitalism?" Transformations of the State. Palgrave Macmillan, London, pp. 3-12. 


\section{Anexo 1 \\ Lista de proyectos}

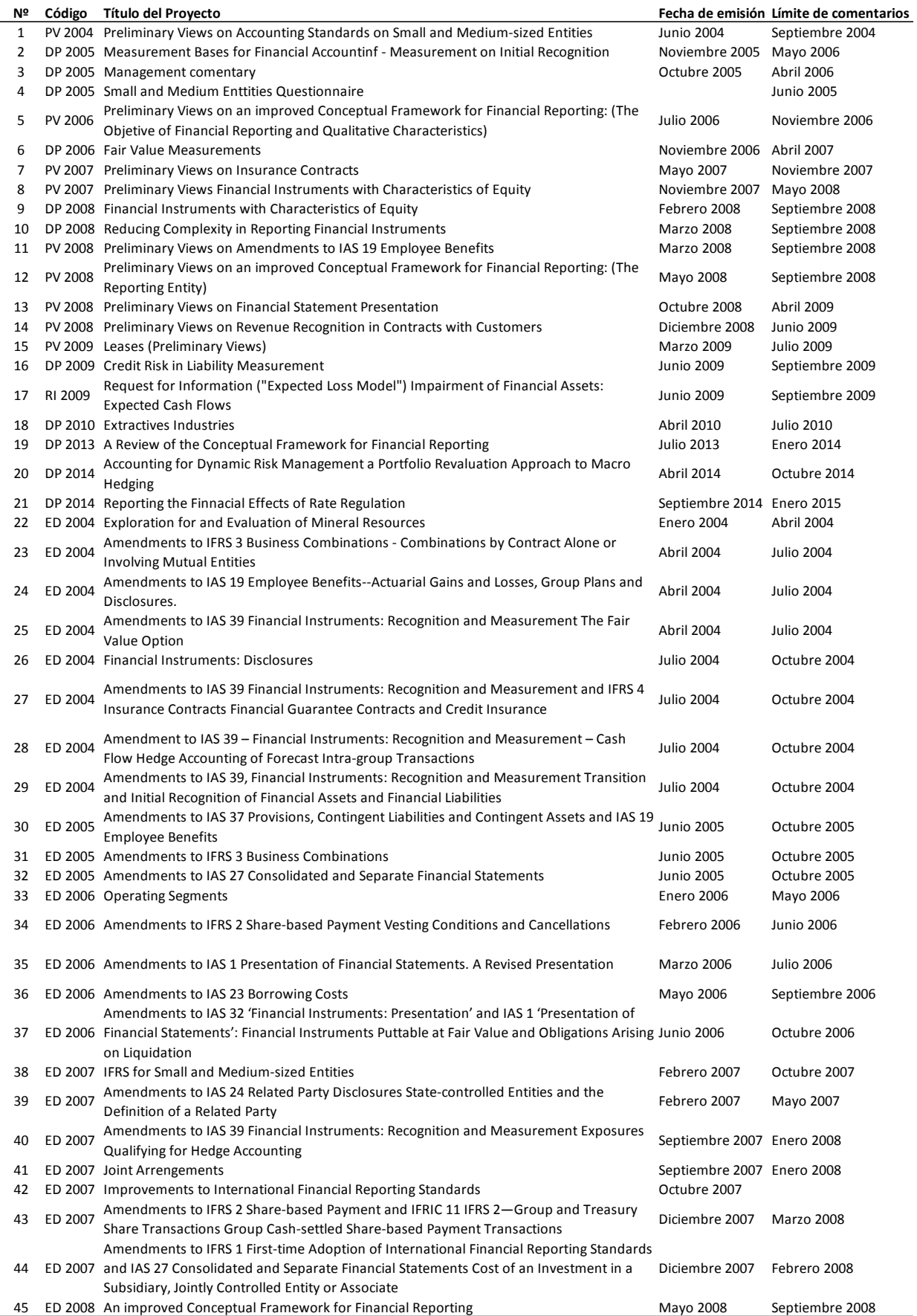


№ Código Título del Proyecto

46 ED 2008 Simplifying Earnings per Share (Proposed amendments to IAS 33)

47 ED 2008 Improvements to IFRS (Proposed amendments to IFRS 1)

48 ED 2008 Additional Exemptions for First-time Adopters

49 ED 2008 Discontinued Operations (Proposed amendments to IFRS 5)

50 ED 2008 Improving Disclosures about Financial Instruments (Proposed amendments to IFRS 7)

51 ED 2008 Relationships with the State (Proposed amendments to IAS 24)

52 ED 2008 Consolidated Financial Statements

53 ED 2008 Embedded Derivatives (Proposed amendments to IFRIC 9 and IAS 39)

54 ED 2008 Investments in Debt Instruments (Proposed amendments to IFRS 7)

55 RC 2008 Review of the Constitution. Identifying issues for Part 2 of the Review

56 ED 2009 Income Tax

57 ED 2009 Derecognition (Proposed amendments to IAS 39 and IFRS 7)

58 ED 2009 Fair Value Measurements

59 ED 2009 Management comentary

60 ED 2009 Financial Instruments: Classification and Measurement

61 ED 2009 Rate-regulated activities

62 ED 2009 Classification of rights Issues (Proposed amendments to IAS 32)

63 ED 2009 Discount Rate for Employee Benefits (Proposed amendments to IAS 19)

64 ED 2009 Improvements to IFRSs. 2008-2010 Cycle

65 DP 2009 Part 2 of the Constitution Review. Proposals for Enhanced Public Accountability

66 ED 2009 Limited Exemption from Comparative IFRS 7 Disclosures for First-time Adopters Proposed

66 ED 2009 amendment to IFRS 1

67 ED 2009 Financial Instruments: Amortised Cost and Impairment

68 ED 2010 Measurement of Liabilities in IAS 37 (Proposed amendments to IAS 37)

69 ED 2010 Conceptual Framework for Financial Reporting. The Reporting Entity

70 ED 2010 Defined Benefit Plans (Proposed amendments to IAS 19)

71 ED 2010 Fair Value Option for Financial Liabilities

Fecha de emisión Límite de comentarios

Agosto 2008 Diciembre 2008

Agosto $2008 \quad$ Noviembre 2008

Septiembre 2008 Enero 2009

Septiembre 2008 Enero 2009

Octubre 2008 Diciembre 2008

Diciembre 2008 Marzo 2009

Diciembre 2008 Marzo 2009

Diciembre 2008 Enero 2009

Diciembre 2008 Enero 2009

Diciembre 2008 Marzo 2009

Marzo 2009 Julio 2009

Abril 2009 Julio 2009

Mayo 2009 Septiembre 2009

Junio 2009 Marzo 2010

Julio $2009 \quad$ Septiembre 2009

Julio $2009 \quad$ Noviembre 2009

Agosto 2009 Septiembre 2009

Agosto 2009 Septiembre 2009

Agosto $2009 \quad$ Noviembre 2009

Septiembre 2009 Noviembre 2009

72 ED 2010 Presentation of Items of Other Comprehensive Income (Proposed amendments to IAS 1)

73 ED 2010 Revenues from Cintracts with Customers

74 ED 2010 Measurement Uncertainty Analysis Disclosure for Fair Value Measurements. Limited re-

exposure of proposed disclosure

75 ED 2010 Insurance Contracts

76 ED 2010 Leases

77 ED 2010 Removal of Fixed Dates for First-time Adopters (Proposed amendments to IFRS 1)

78 ED 2010 Deferred Tax: Recovery of Underlying Assets (Proposed amendments to IAS 12)

79 ED 2010 Severe Hyperinflation (Proposed amendment to IFRS 1)

80 PC 2010 Status of Trustees' Strategy Review

81 ED 2010 Hedge Accounting

82 ED 2011 Financial Instruments: Impairment

83 ED 2011 Offsetting Financial Assets and Financial Liabilities

Noviembre 2009 Diciembre 2009

Noviembre 2009 Junio 2010

Enero $2010 \quad$ Abril 2010

Marzo $2010 \quad$ Julio 2010

Abril 2010 Septiembre 2010

Mayo $2010 \quad$ Julio

84 RT 2011 Report of the Trustees' Strategy Review IFRSs as the Global Standard: Setting a Strategy fo

85 ED 2011 Improvements to IFRSs. 2009-2011 Cycle

86 ED 2011 Mandatory Effective Date of IFRS 9

87 ED 2011 Investment Entities

88 ED 2011 Government Loans (Proposed amendments to IFRS 1)

89 ED 2011 Revenue from Contracts with Customers

90 ED 2011 Transition Guidance Proposed amendments to IFRS 10

91 ED 2012 Invitation to Comment IASB and IFRS Interpretations Committee Due Process Handbook

92 ED 2012 Annual Improvements to IFRSs. 2010-2012 Cycle

93 RI 2012 Request for Information. Comprehensive Review of the IFRS for SMEs

94 RI 2012 Request for Information. Post-implementation Review: IFRS 8 Operating Segments

95 ED 2012 Annual Improvements to IFRSs. 2011-2013 Cycle

96 ED 2012 Equity Method: Share of Other Net Asset Changes (Proposed amendments to IAS 28)

97 ED 2012 Classification and Measurement: Limited Amendments to IFRS 9. Proposed amendments

97 ED 2012 to IFRS 9 (2010)

98 ED 2012 Clarification of Acceptable Methods of Depreciation and Amortisation (Proposed amendments to IAS 16 and IAS 38

Mayo 2010

Septiembre 2010

Junio 2010 Octubre 2010

Junio 2010

Septiembre 2010

Julio 2010

Noviembre 2010

Agosto 2010 Diciembre 2010

Agosto $2010 \quad$ Octubre 2010

Septiembre 2010 Noviembre 2010

Septiembre 2010 Noviembre 2010

Noviembre 2010 Febrero 2011

Diciembre 2010 Marzo 2011

Enero $2011 \quad$ Abril 2011

Enero $2011 \quad$ Abril 2011

Abril 2011 Julio 2011

Junio $2011 \quad$ Octubre 2011

Agosto $2011 \quad$ Octubre 2011

Agosto 2011 Enero 2012

Octubre 2011 Enero 2012

Noviembre 2011 Marzo 2012

Diciembre 2011 Marzo 2012

Mayo 2012 Septiembre 2012

Mayo 2012 Septiembre 2012

Junio $2012 \quad$ Noviembre 2012

Julio $2012 \quad$ Noviembre 2012

Noviembre 2012 Febrero 2013

Noviembre 2012 Marzo 2013

Noviembre 2012 Marzo 2013

Diciembre 2012 Abril 2013

Diciembre 2012 Abril 2013

Diciembre 2012 Abril 2013

100 ED 2012 Acquisition of an Interest in a Joint Operation (Proposed amendment to IFRS 11)

101 Recoverable Amount Disclosures for Non-Financial Assets (Proposed Amendments to IAS 36)

Enero 2013

Marzo 2013 


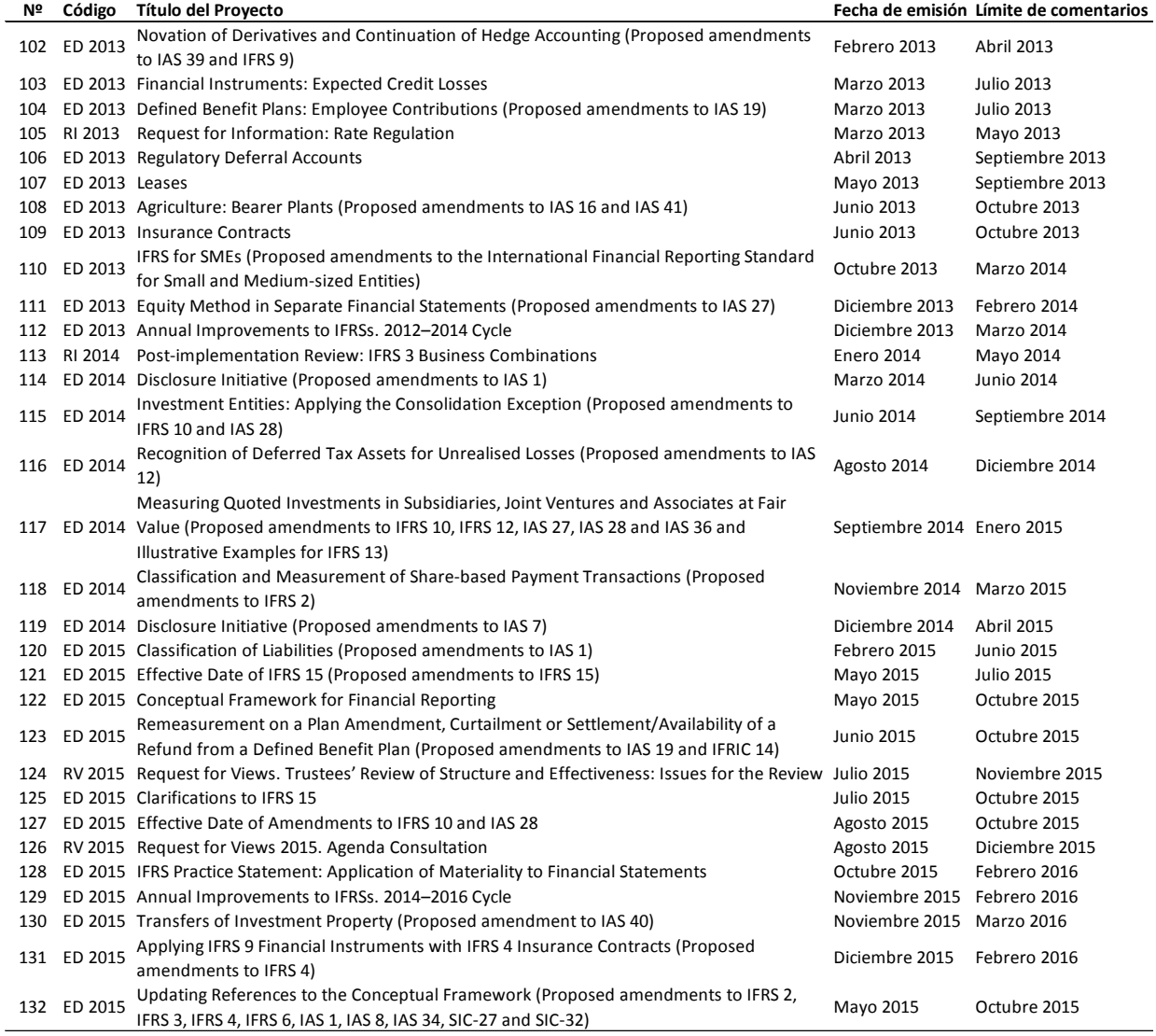

Fuente: Fundación IFRS y elaboración propia. 